\title{
The homogeneous pseudo-embeddings and hyperovals of the generalized quadrangle $H(3,4)$
}

\author{
Bart De Bruyn and Mou Gao
}

January 30, 2020

\begin{abstract}
In this paper, we determine all homogeneous pseudo-embeddings of the generalized quadrangle $H(3,4)$ and give a description of all its even sets. Using this description, we subsequently compute all hyperovals of $H(3,4)$, up to isomorphism, and give computer free descriptions of them. Several of these hyperovals, but not all of them, have already been described before in the literature.
\end{abstract}

MSC2010: 51E12, 51A45, 20C20, 94B05

Key words: generalized quadrangle, even set, hyperoval, (homogeneous) pseudo-embedding, pseudo-hyperplane

\section{Introduction}

Suppose $\mathcal{S}=(\mathcal{P}, \mathcal{L}, \mathrm{I})$ is a point-line geometry with the property that the number of points on each line is finite and at least three. A pseudo-embedding of $\mathcal{S}$ is a mapping $\epsilon$ from the point set $\mathcal{P}$ of $\mathcal{S}$ to the set of points of a projective space $\mathrm{PG}(V)$, with $V$ an $\mathbb{F}_{2}$-vector space, such that the following two properties are satisfied:

- the image $\epsilon(\mathcal{P})$ of $\epsilon$ generates $\mathrm{PG}(V)$;

- if $L$ is a line with points $x_{1}, x_{2}, \ldots, x_{k}$, then $\epsilon\left(x_{1}\right), \epsilon\left(x_{2}\right), \ldots, \epsilon\left(x_{k}\right)$ is a frame of the subspace $\langle\epsilon(L)\rangle$ of $\mathrm{PG}(V)$, i.e. there exist linearly independent vectors $\bar{v}_{1}, \bar{v}_{2}, \ldots, \bar{v}_{k-1}$ of $V$ such that $\epsilon\left(x_{i}\right)=\left\langle\bar{v}_{i}\right\rangle, \forall i \in\{1,2, \ldots, k-1\}$, and $\epsilon\left(x_{k}\right)=\left\langle\bar{v}_{1}+\bar{v}_{2}+\cdots+\bar{v}_{k-1}\right\rangle$.

We often denote such a pseudo-embedding by $\epsilon: \mathcal{S} \rightarrow \mathrm{PG}(V)$. Two pseudo-embeddings $\epsilon_{1}: \mathcal{S} \rightarrow \mathrm{PG}\left(V_{1}\right)$ and $\epsilon_{2}: \mathcal{S} \rightarrow \mathrm{PG}\left(V_{2}\right)$ of $\mathcal{S}$ are called isomorphic if there exists a linear isomorphism $\theta$ from $V_{1}$ to $V_{2}$ such that $\epsilon_{2}=\theta \circ \epsilon_{1}$.

A pseudo-embedding $\epsilon: \mathcal{S} \rightarrow \mathrm{PG}(V)$ is called $G$-homogeneous for some group $G$ of automorphisms of $\mathcal{S}$ if for every $\theta \in G$, there exists a (necessarily unique) $\widetilde{\theta} \in G L(V)$ such that $\widetilde{\theta} \circ \epsilon=\epsilon \circ \theta$. The map $G \rightarrow G L(V): \theta \mapsto \tilde{\theta}$ then defines a modular representation 
of $G$, and $V$ becomes a $G$-module. The pseudo-embedding $\epsilon$ is called homogeneous if it is $\operatorname{Aut}(\mathcal{S})$-homogeneous with $\operatorname{Aut}(S)$ the full group of automorphisms of $\mathcal{S}$.

Suppose $\epsilon: \mathcal{S} \rightarrow \mathrm{PG}(V)$ is a pseudo-embedding of $\mathcal{S}$ and $\alpha$ is a subspace of $\mathrm{PG}(V)$ disjoint from $\epsilon(\mathcal{P})$ and all subspaces $\langle\epsilon(L)\rangle$, where $L \in \mathcal{L}$. Then the map $x \mapsto\langle\alpha, \epsilon(x)\rangle$ defines a pseudo-embedding $\epsilon / \alpha$ of $\mathcal{S}$ into the quotient projective space $\mathrm{PG}(V) / \alpha$ (whose points are the subspaces of $\mathrm{PG}(V)$ that contain $\alpha$ as a hyperplane). We call $\epsilon / \alpha$ a quotient of $\epsilon$. If $\epsilon_{1}, \epsilon_{2}$ are two pseudo-embeddings of $\mathcal{S}$, then we write $\epsilon_{1} \geq \epsilon_{2}$ if $\epsilon_{2}$ is isomorphic to a quotient of $\epsilon_{1}$.

If $\widetilde{\epsilon}$ is a pseudo-embedding of $\mathcal{S}$ such that $\widetilde{\epsilon} \geq \epsilon$ for any other pseudo-embedding $\epsilon$ of $\mathcal{S}$, then $\widetilde{\epsilon}$ is called a universal pseudo-embedding. If $\mathcal{S}$ has pseudo-embeddings, then it also has a universal pseudo-embedding which is moreover unique, up to isomorphism. The universal pseudo-embedding is always homogeneous. The vector dimension of the universal pseudo-embedding is called the pseudo-embedding rank. In case $|\mathcal{P}|<\infty$, the pseudo-embedding rank is equal to $|\mathcal{P}|-\operatorname{dim}(C)$, where $C$ is the binary code of length $|\mathcal{P}|$ generated by the characteristic vectors of the lines of $\mathcal{S}$.

Pseudo-embeddings were introduced in [9] and further investigated in [8, 10]. We refer to these papers for proofs of the above-mentioned facts. As mentioned above, there exist connections between pseudo-embeddings and certain binary codes. The various results obtained in the papers [8, 9, 10] offer extra tools by which these binary codes can be studied. There also exist connections between $G$-homogeneous pseudo-embeddings and $\mathbb{F}_{2}$-representations for $G$. In practice, many of these $\mathbb{F}_{2}$-representations turn out to be reducible. Also, given an $\mathbb{F}_{2}$-representation of $G$, there is not necessarily an associated $G$-homogeneous pseudo-embedding, and if there is one, there might not be a natural way to decide which orbit(s) of the $G$-module correspond(s) to the points of the geometry. It is possible that $G$ occurs as automorphism group of two non-isomorphic geometries and that a certain $G$-module hosts a $G$-homogeneous pseudo-embedding for only one of them. For studying $G$-homogeneous pseudo-embeddings, the group $G$ does therefore not tell the whole story, and the underlying geometric structure of the geometry should still be taken into account. In this paper, we classify all homogeneous pseudo-embeddings of a particular generalized quadrangle, and give some applications of this classification.

A point-line geometry $\mathcal{S}=(\mathcal{P}, \mathcal{L}, \mathrm{I})$ is called a generalized quadrangle [17] if every point is incident with at least two lines and if for every non-incident point-line pair $(x, L)$, there exists a unique point on $L$ collinear with $x$. A generalized quadrangle is said to have $\operatorname{order}(s, t)$ if every line is incident with precisely $s+1$ points and if every point is incident with exactly $t+1$ lines. The point-line dual of any generalized quadrangle is again a generalized quadrangle. A standard counting yields that a generalized quadrangle of order $(s, t)$ contains $(s+1)(s t+1)$ points and $(t+1)(s t+1)$ lines.

Consider now in $\mathrm{PG}(3,4)$ the Hermitian surface $\mathcal{H}$ with equation $X_{1} X_{2}^{2}+X_{2} X_{1}^{2}+$ $X_{3} X_{4}^{2}+X_{4} X_{3}^{2}=0$. The points and lines of $\mathrm{PG}(3,4)$ contained in $\mathcal{H}$ then define a generalized quadrangle of order $(4,2)$ on 45 points which we will denote by $H(3,4)$. In this paper, we determine the pseudo-embedding rank and all homogeneous pseudo-embeddings of $H(3,4)$. Our results are as follows. 
Theorem 1.1. The pseudo-embedding rank of $H(3,4)$ is equal to 24 . As a consequence, the dimension of the binary code generated by the characteristic vectors of the lines of $H(3,4)$ has dimension $45-24=21$.

We also show that there are up to isomorphism four homogeneous pseudo-embeddings of $H(3,4)$, with respective vector dimensions $14,15,23$ and 24 . In order to describe these, we need to introduce a number of notations. We put $\mathbb{F}_{2}=\{0,1\} \subseteq \mathbb{F}_{4}=\left\{0,1, \omega, \omega^{2}\right\}$, and consider a 24 -dimensional $\mathbb{F}_{2}$-vector space $V_{24}$ with a basis $B$ consisting of the following vectors:

- $\bar{g}_{1}, \bar{g}_{2}, \bar{g}_{3}, \bar{g}_{4}, \bar{h}_{34}, \bar{k}$

- $\bar{g}_{12}, \bar{h}_{12}, \bar{g}_{13}, \bar{h}_{13}, \bar{g}_{14}, \bar{h}_{14}, \bar{g}_{23}, \bar{h}_{23}, \bar{g}_{24}, \bar{h}_{24}$,

- $\bar{g}_{123}, \bar{h}_{123}, \bar{g}_{124}, \bar{h}_{124}, \bar{g}_{134}, \bar{h}_{134}, \bar{g}_{234}, \bar{h}_{234}$.

Consider in $V_{24}$ the following subspaces:

- $V_{23}$ is generated by the vectors of $B \backslash\{\bar{k}\}$;

- $V_{15}$ is generated by the vectors of $B \backslash\left\{\bar{k}, \bar{g}_{123}, \bar{h}_{123}, \ldots, \bar{g}_{234}, \bar{h}_{234}\right\}$;

- $V_{14}$ is generated by the vectors of $B \backslash\left\{\bar{k}, \bar{h}_{34}, \bar{g}_{123}, \bar{h}_{123}, \ldots, \bar{g}_{234}, \bar{h}_{234}\right\}$.

Consider also the following summations:

- $\Sigma_{1}$ : summation over all $i \in\{1,2,3,4\}$;

- $\Sigma_{2}$ : summation over all $i, j \in\{1,2,3,4\}$ with $i<j$;

- $\Sigma_{2^{\prime}}$ : summation over all $i, j \in\{1,2,3,4\}$ with $i<j$ and $(i, j) \neq(3,4)$;

- $\Sigma_{3}$ : summation over all $i, j, k \in\{1,2,3,4\}$ with $i<j<k$.

We consider now four maps from $\mathcal{H}$ to the point sets of certain projective spaces. The map $\epsilon_{24}$ maps the point $\left(X_{1}, X_{2}, X_{3}, X_{4}\right) \in \mathcal{H}$ to the point of $\mathrm{PG}\left(V_{24}\right)$ that is generated by the vector

$$
\begin{aligned}
& \left(\omega X_{3} X_{4}^{2}+\omega^{2} X_{4} X_{3}^{2}\right) \bar{h}_{34}+\left(\left(X_{1}^{3}+X_{2}^{3}+X_{1}^{3} X_{2}^{3}\right)\left(X_{3}^{3}+X_{4}^{3}+X_{3}^{3} X_{4}^{3}\right)+1\right) \bar{k} \\
& \quad+\sum_{1} X_{i}^{3} \bar{g}_{i}+\sum_{2^{\prime}}\left(\left(X_{i} X_{j}^{2}+X_{j} X_{i}^{2}\right) \bar{g}_{i j}+\left(\omega X_{i} X_{j}^{2}+\omega^{2} X_{j} X_{i}^{2}\right) \bar{h}_{i j}\right) \\
& +\sum_{3}\left(\left(X_{i} X_{j} X_{k}+X_{i}^{2} X_{j}^{2} X_{k}^{2}\right) \bar{g}_{i j k}+\left(\omega X_{i} X_{j} X_{k}+\omega^{2} X_{i}^{2} X_{j}^{2} X_{k}^{2}\right) \bar{h}_{i j k}\right) .
\end{aligned}
$$

Note that the latter is indeed a vector of $V_{24}$ as $a^{3}, b+b^{2} \in \mathbb{F}_{2}$ for all $a, b \in \mathbb{F}_{4}$. The map $\epsilon_{23}$ maps the point $\left(X_{1}, X_{2}, X_{3}, X_{4}\right) \in \mathcal{H}$ to the point of $\mathrm{PG}\left(V_{23}\right)$ that is generated by the vector

$$
\begin{aligned}
\left(\omega X_{3} X_{4}^{2}+\right. & \left.\omega^{2} X_{4} X_{3}^{2}\right) \bar{h}_{34}+\sum_{1} X_{i}^{3} \bar{g}_{i}+\sum_{2^{\prime}}\left(\left(X_{i} X_{j}^{2}+X_{j} X_{i}^{2}\right) \bar{g}_{i j}+\left(\omega X_{i} X_{j}^{2}+\omega^{2} X_{j} X_{i}^{2}\right) \bar{h}_{i j}\right) \\
& +\sum_{3}\left(\left(X_{i} X_{j} X_{k}+X_{i}^{2} X_{j}^{2} X_{k}^{2}\right) \bar{g}_{i j k}+\left(\omega X_{i} X_{j} X_{k}+\omega^{2} X_{i}^{2} X_{j}^{2} X_{k}^{2}\right) \bar{h}_{i j k}\right) .
\end{aligned}
$$

The map $\epsilon_{15}$ maps the point $\left(X_{1}, X_{2}, X_{3}, X_{4}\right) \in \mathcal{H}$ to the point of $\mathrm{PG}\left(V_{15}\right)$ that is generated by the vector

$$
\left(\omega X_{3} X_{4}^{2}+\omega^{2} X_{4} X_{3}^{2}\right) \bar{h}_{34}+\sum_{1} X_{i}^{3} \bar{g}_{i}+\sum_{2^{\prime}}\left(\left(X_{i} X_{j}^{2}+X_{j} X_{i}^{2}\right) \bar{g}_{i j}+\left(\omega X_{i} X_{j}^{2}+\omega^{2} X_{j} X_{i}^{2}\right) \bar{h}_{i j}\right) .
$$


The map $\epsilon_{14}$ maps the point $\left(X_{1}, X_{2}, X_{3}, X_{4}\right) \in \mathcal{H}$ to the point of $\mathrm{PG}\left(V_{14}\right)$ that is generated by the vector

$$
\left(\omega X_{3} X_{4}^{2}+\omega^{2} X_{4} X_{3}^{2}\right) \bar{h}_{12}+\sum_{1} X_{i}^{3} \bar{g}_{i}+\sum_{2^{\prime}}\left(\left(X_{i} X_{j}^{2}+X_{j} X_{i}^{2}\right) \bar{g}_{i j}+\left(\omega X_{i} X_{j}^{2}+\omega^{2} X_{j} X_{i}^{2}\right) \bar{h}_{i j}\right) .
$$

We will prove the following.

Theorem 1.2. Up to isomorphism, $H(3,4)$ has four homogeneous pseudo-embeddings. Specifically, every pseudo-embedding of $H(3,4)$ is isomorphic to either $\epsilon_{24}, \epsilon_{23}, \epsilon_{15}$ or $\epsilon_{14}$. The pseudo-embedding $\epsilon_{24}$ is universal.

An even set of a point-line geometry is a set of points meeting each line in an even number of points. The empty point set is the trivial example of an even set. A nontrivial even set intersecting each line in either 0 or 2 points is called a hyperoval. Hyperovals of generalized quadrangles play a fundamental role in the study of the so-called extended generalized quadrangles, see e.g. [3, 13, 14, 15]. The more general notion of a hyperoval (sometimes also called a local subspace) of a polar space has also been studied; these objects first arose in [2] in view of their connection with so-called locally polar spaces.

The complements of the nontrivial even sets are also called pseudo-hyperplanes. If $\mathcal{S}=(\mathcal{P}, \mathcal{L}, \mathrm{I})$ is a point-line geometry for which the number of points on each line is finite and at least three such that $\mathcal{S}$ has a pseudo-embedding, then by [9, Theorem 1.3] all pseudo-hyperplanes of $\mathcal{S}$ have the form $H_{\Pi}:=\widetilde{\epsilon}^{-1}(\widetilde{\epsilon}(\mathcal{P}) \cap \Pi)$, where $\widetilde{\epsilon}: \mathcal{S} \rightarrow \operatorname{PG}(\widetilde{V})$ is the universal pseudo-embedding of $\mathcal{S}$ and $\Pi$ is a hyperplane of $\mathrm{PG}(\widetilde{V})$. The correspondence $\Pi \leftrightarrow H_{\Pi}$ is moreover bijective. In view of Theorem 1.2 , we thus have the following.

Corollary 1.3. The even sets of $H(3,4)$ are precisely the subsets of $\mathcal{H}$ satisfying an equation of the form

$$
\begin{gathered}
\sum_{1}\left(a_{i} X_{i}^{3}\right)+a_{5}\left(\omega X_{3} X_{4}^{2}+\omega^{2} X_{4} X_{3}^{2}\right)+a_{6}\left(\left(X_{1}^{3}+X_{2}^{3}+X_{1}^{3} X_{2}^{3}\right)\left(X_{3}^{3}+X_{4}^{3}+X_{3}^{3} X_{4}^{3}\right)+1\right) \\
+\sum_{2^{\prime}}\left(b_{i j} X_{i} X_{j}^{2}+b_{i j}^{2} X_{j} X_{i}^{2}\right)+\sum_{3}\left(b_{i j k} X_{i} X_{j} X_{k}+b_{i j k}^{2} X_{i}^{2} X_{j}^{2} X_{k}^{2}\right)=1
\end{gathered}
$$

with the $a_{i}$ 's belonging to $\mathbb{F}_{2}$ and the $b_{i j}$ 's and $b_{i j k}$ 's belonging to $\mathbb{F}_{4}$.

There are thus $2^{24}$ even sets in $H(3,4)$. In fact, Corollary 1.3 gives a bijective correspondence between the elements $\bar{a}=\left(a_{1}, \ldots, a_{6}, b_{12}, \ldots, b_{24}, b_{123}, \ldots, b_{234}\right) \in \mathbb{F}_{2}^{6} \times \mathbb{F}_{4}^{9}$ and the even sets $E(\bar{a})$ of $H(3,4)$. With the aid of a computer, we will determine for which $\bar{a} \in \mathbb{F}_{2}^{6} \times \mathbb{F}_{4}^{9}, E(\bar{a})$ is a hyperoval. We also investigate when two elements $\bar{a}_{1}, \bar{a}_{2} \in \mathbb{F}_{2}^{6} \times \mathbb{F}_{4}^{9}$ give rise to isomorphic even sets $E\left(\bar{a}_{1}\right)$ and $E\left(\bar{a}_{2}\right)$. This allows us to conclude the following.

Theorem 1.4. The generalized quadrangle $H(3,4)$ has 70648 hyperovals which fall into 23 isomorphism classes. 
Although many of these hyperovals have already been described before in the literature, several of them also appear to be new. In fact, for each construction mentioned in the literature we will indicate with which of the 23 hyperovals it corresponds. Based on Corollary 1.3, we also give explicit unified descriptions for representatives of the various isomorphism classes of hyperovals. In order to keep these descriptions as simple as possible, we have chosen representatives for which the corresponding elements $\bar{a} \in \mathbb{F}_{2}^{6} \times \mathbb{F}_{4}^{9}$ have smallest possible weights.

We will also determine some properties of the hyperovals. This will make identification easier each time a hyperoval of $H(3,4)$ emerges somewhere. One of these properties is a description of the 3-regular subgraph of the collinearity graph induced on the hyperoval. In fact, these regular graphs were also classified in [13] (up to isomorphism) for the hyperovals on $6,8,10,12$ and 14 vertices. Our classification of the hyperovals shows that some 3-regular graphs on 12 and 14 vertices are missing in the discussion in [13] (and others do not correspond to hyperovals). We will see that it is also possible that nonisomorphic hyperovals can have isomorphic underlying 3-regular subgraphs. Note that a list of the 3-regular subgraphs that can occur as induced subgraphs of the hyperovals gives important structural information about the hyperovals, but not at all a complete classification.

\section{Enlarging pseudo-embeddings}

Let $\mathcal{S}=(\mathcal{P}, \mathcal{L}, \mathrm{I})$ be a point-line geometry with the property that the number of points on each line is finite and at least three. Suppose $\mathcal{S}$ has a pseudo-embedding $\epsilon: \mathcal{S} \rightarrow \operatorname{PG}(V)$. Then for every hyperplane $\Pi$ of $\mathrm{PG}(V)$, the set $H_{\Pi}:=\epsilon^{-1}(\epsilon(\mathcal{P}) \cap \Pi)$ is a pseudo-hyperplane of $\mathcal{S}$. We will say that $H_{\Pi}$ arises from $\epsilon$. We denote by $\mathcal{H}_{\epsilon}$ the set of all pseudo-hyperplanes that arise from $\epsilon$. If $\mathcal{S}$ has pseudo-embeddings and $\widetilde{\epsilon}: \mathcal{S} \rightarrow \operatorname{PG}(\widetilde{V})$ denotes its universal pseudo-embedding, then as mentioned before $\mathcal{H}_{\widetilde{\epsilon}}$ is the set of all pseudo-hyperplanes.

Under certain circumstances, it is possible to modify an existing pseudo-embedding to obtain a "larger" pseudo-embedding. This procedure is explained in the following proposition.

Proposition 2.1. Let $\epsilon: \mathcal{S} \rightarrow \Sigma$ be a pseudo-embedding of $\mathcal{S}$ and suppose $H$ is a pseudohyperplane of $\mathcal{S}$ not arising from $\epsilon$. Embed $\Sigma$ as a hyperplane in $\bar{\Sigma}$, and let $p \in \bar{\Sigma} \backslash \Sigma$. For every point $x \in H$, we define $\bar{\epsilon}(x):=\epsilon(x)$ and for every point $y$ of $\mathcal{S}$ not contained in $H$, let $\bar{\epsilon}(y)$ denote the third point on the line $\epsilon(y) p$. Then $\bar{\epsilon}: \mathcal{S} \rightarrow \bar{\Sigma}$ is a pseudo-embedding of $\mathcal{S}$.

Proof. Put $\Sigma=\mathrm{PG}(V)$ and $\bar{\Sigma}=\mathrm{PG}(\bar{V})$, where $V$ is a hyperplane of the $\mathbb{F}_{2}$-vector space $\bar{V}$. Let $\bar{v}^{*} \in \bar{V}$ such that $p=\left\langle\bar{v}^{*}\right\rangle$. Let $L=\left\{x_{1}, x_{2}, \ldots, x_{k}\right\}$ be an arbitrary line of $\mathcal{S}$. Without loss of generality, we may suppose that there exists an $l \in\left\{0,1, \ldots,\left\lfloor\frac{k}{2}\right\rfloor\right\}$ such that $x_{1}, x_{2}, \ldots, x_{k-2 l} \in H$ and $x_{k-2 l+1}, \ldots, x_{k} \notin H$. Let $\bar{v}_{1}, \bar{v}_{2}, \ldots, \bar{v}_{k} \in V$ such that $\epsilon\left(x_{i}\right)=\left\langle\bar{v}_{i}\right\rangle$ for every $i \in\{1,2, \ldots, k\}$. For every $i \in\{1,2, \ldots, k-2 l\}$, we put $\bar{v}_{i}^{\prime}:=\bar{v}_{i}$, and for every $i \in\{k-2 l+1, \ldots, k\}$, we put $\bar{v}_{i}^{\prime}=\bar{v}_{i}+\bar{v}^{*}$. Then $\bar{\epsilon}\left(x_{i}\right)=\left\langle\bar{v}_{i}^{\prime}\right\rangle$ for every 
$i \in\{1,2, \ldots, k\}$. We have $\bar{v}_{1}^{\prime}+\bar{v}_{2}^{\prime}+\cdots+\bar{v}_{k}^{\prime}=\bar{v}_{1}+\bar{v}_{2}+\cdots+\bar{v}_{k}+2 l \cdot \bar{v}^{*}=\bar{v}_{1}+\bar{v}_{2}+\cdots+\bar{v}_{k}=\bar{o}$. Moreover, since the vectors $\bar{v}_{1}, \bar{v}_{2}, \ldots, \bar{v}_{k-1}$ are linearly independent, also $\bar{v}_{1}^{\prime}, \bar{v}_{2}^{\prime}, \ldots, \bar{v}_{k-1}^{\prime}$ are linearly independent. So, $\bar{\epsilon}$ is a pseudo-embedding of $\mathcal{S}$ in a suitable subspace of $\bar{\Sigma}$.

We still need to show that the image of $\bar{\epsilon}$ generates the whole projective space $\bar{\Sigma}$. Suppose the image is contained in a hyperplane $\Pi$. Then $\Pi \neq \Sigma$ and so $\Pi^{\prime}:=\Pi \cap \Sigma$ is a hyperplane of $\Sigma$. If $\Pi$ contains $p$, then every point of the image of $\bar{\epsilon}$ not contained in $\Sigma$ lies on a line joining $p$ with a point of $\Pi^{\prime}$, implying that the image of $\epsilon$ is contained in $\Pi^{\prime}$. This is impossible. So, $p \notin \Pi$ and $\Pi$ is the unique hyperplane through $\Pi^{\prime}$ distinct from $\left\langle p, \Pi^{\prime}\right\rangle$ and $\Sigma$. Now, $\bar{\epsilon}$ maps the points of $H$ to points of $\Pi^{\prime}$ and the points not in $H$ to points of $\Pi \backslash \Pi^{\prime}$. This implies that $\epsilon$ maps points of $H$ to points of $\Pi^{\prime}$ and points not in $H$ to points of $\Sigma \backslash \Pi^{\prime}$. But that is impossible as $H$ does not arise from $\epsilon$.

\section{An upper bound for the pseudo-embedding rank of $H(3,4)$}

Not all point-line geometries with the property that the number of points on each line is finite and at least three have pseudo-embeddings. In [9] (see Corollary 3.11(1)), it was however shown that every finite generalized quadrangle of order $(s, t), s \geq 2$, admits pseudo-embeddings. In particular, this applies to the generalized quadrangle $H(3,4)$. In this section, we show that the pseudo-embedding rank of $H(3,4)$ is at most 24 . This upper bound is essential for the treatment given in Section 4, where we will show among other things that the pseudo-embedding rank is precisely 24 .

Again, let $\mathcal{S}=(\mathcal{P}, \mathcal{L}, \mathrm{I})$ be a point-line geometry with the property that the number of points on each line is finite and at least three. A pseudo-subspace of $\mathcal{S}$ is a set $S$ of points with the property that no line $L$ intersects the complement of $S$ in a singleton, i.e. if there are $k$ points on $L$ and $k-1$ of these points are known to belong to $S$ then also the remaining point must belong to $S$. The whole point set $\mathcal{P}$ is an example of a pseudo-subspace, and the intersection of any number of pseudo-subspaces is again a pseudo-subspace. So, given a nonempty set of points $X$, the intersection $[X]$ of all pseudo-subspaces containing $X$ is the smallest pseudo-subspace that contains $X$. If $[X]$ coincides with the whole point set, then $X$ is called a pseudo-generating set. The smallest size of a pseudo-generating set is called the pseudo-generating rank. The following proposition is precisely Theorem 1.5 of [9].

Proposition 3.1. Suppose $\mathcal{S}$ has pseudo-embeddings. Then the following hold.

(1) The pseudo-embedding rank of $\mathcal{S}$ is bounded above by the pseudo-generating rank of $\mathcal{S}$.

(2) If there exists a pseudo-embedding $\epsilon: \mathcal{S} \rightarrow \mathrm{PG}(V)$ and a pseudo-generating set $X$ of $\mathcal{S}$ such that $|X|=\operatorname{dim}(V)<\infty$, then the pseudo-embedding and pseudogenerating ranks of $\mathcal{S}$ are equal to $\operatorname{dim}(V)$ and $\epsilon$ is isomorphic to the universal pseudo-embedding of $\mathcal{S}$. 
If $x$ is a point of a generalized quadrangle $\mathcal{Q}$, then $x^{\perp}$ denotes the set of points of $\mathcal{Q}$ equal to or collinear with $x$. If $X$ is a nonempty set of points of $\mathcal{Q}$, then we define $X^{\perp}:=\bigcap_{x \in X} x^{\perp}$.

Lemma 3.2. $H(3,4)$ has a pseudo-generating set of size 24 .

Proof. We will reason in the dual generalized quadrangle $H^{D}(3,4)$ of $H(3,4)$. We recall some facts about this dual generalized quadrangle taken from [17]. The generalized quadrangle $H^{D}(3,4)$ is isomorphic to the generalized quadrangle $Q(5,2)$ of the points and lines of an elliptic quadric in the projective space $\mathrm{PG}(5,2)$. Its order is $(2,4)$ and it contains a sub(generalized)quadrangle $Q(4,2)$ of order $(2,2)$. If $x$ is a point of $Q(5,2)$ not belonging to $Q(4,2)$, then $O_{x}:=x^{\perp} \cap Q(4,2)$ is an ovoid of $Q(4,2)$, i.e. a set of five points meeting each line in a singleton. We call $O_{x}$ the ovoid of $Q(4,2)$ subtended by the point $x$. The generalized quadrangle $Q(4,2)$ has six ovoids, which two by two intersect in a singleton. Each of these ovoids is subtended by exactly two points of $Q(5,2) \backslash Q(4,2)$. Every point $x$ of $Q(4,2)$ is contained in precisely two ovoids. If $\left\{x, y_{1}, y_{2}\right\}$ is a line of $Q(5,2)$ through $x$ not contained in $Q(4,2)$, then these ovoids are precisely $y_{1}^{\perp} \cap Q(4,2)$ and $y_{2}^{\perp} \cap Q(4,2)$.

Now, let $O$ be an ovoid of $Q(4,2)$ and $L$ a line of $Q(4,2)$. Put $\left\{x^{*}\right\}=L \cap O$. Let $\mathcal{L}_{1}$ be the set of 14 lines of $Q(4,2)$ distinct from $L$ and let $\mathcal{L}_{2}$ be a set of 10 lines of $Q(5,2)$ not contained in $Q(4,2)$ such that each of the 10 points of $Q(4,2) \backslash O$ is contained in a unique line of $\mathcal{L}_{2}$. We show that the 24-set $\mathcal{L}_{1} \cup \mathcal{L}_{2}$ is a pseudo-generating set of the point-line dual $Q^{D}(5,2) \cong H(3,4)$ of $Q(5,2)$. Let $\mathcal{L}$ denote the smallest pseudo-subspace of $Q^{D}(5,2)$ containing $\mathcal{L}_{1} \cup \mathcal{L}_{2}$.

Each of the eight points of $Q(4,2) \backslash(L \cup O)$ is contained in three lines of $\mathcal{L}_{1}$ and one line of $\mathcal{L}_{2}$, and since $\mathcal{L}$ is a pseudo-subspace, all five lines through that point belong to $\mathcal{L}$.

Now, let $O^{\prime}$ be the unique ovoid of $Q(4,2)$ through $x^{*}$ distinct from $O$. This ovoid $O^{\prime}$ is subtended by two points $u_{1}$ and $u_{2}$. Then $x^{*} u_{1}$ and $x^{*} u_{2}$ are the two lines of $Q(5,2)$ through $x^{*}$ not contained in $Q(4,2)$. The four lines through $u_{i}, i \in\{1,2\}$, meeting $\left(O^{\prime} \backslash L\right) \subseteq(Q(4,2) \backslash(L \cup O))$ are contained in $\mathcal{L}$ and so also the fifth line through $u_{i}$ also belongs to $\mathcal{L}$. This implies that all two lines of $Q(5,2)$ through $x^{*}$ not contained in $Q(4,2)$ belong to $\mathcal{L}$. As there are also two lines of $\mathcal{L}_{1} \subseteq \mathcal{L}$ through $x^{*}$, we see that the fifth line $L$ through $x^{*}$ also belongs to $\mathcal{L}$. Every point of $L \backslash\left\{x^{*}\right\}$ is thus contained in three lines of $\mathcal{L}$ that are in $Q(4,2)$ and one line of $\mathcal{L}_{2} \subseteq \mathcal{L}$, implying that also the fifth line through that point belongs to $\mathcal{L}$.

The above allows to conclude that all lines of $Q(4,2)$ as well as all lines intersecting $Q(4,2)$ in a point not contained in $O$ belong to $\mathcal{L}$.

We now show that also every line $K$ meeting $Q(4,2)$ in a point $x \in O$ belongs to $\mathcal{L}$. Take to that end the point $w \in K \backslash\{x\}$ such that $w^{\perp} \cap Q(4,2) \neq O$. Then the four lines through $w$ distinct from $K$ belong to $\mathcal{L}$, implying that also $K$ must belong to $\mathcal{L}$.

The following is a consequence of Proposition 3.1(1) and Lemma 3.2 .

Corollary 3.3. The pseudo-embedding and pseudo-generating ranks of $H(3,4)$ are at most 24. 


\section{Three homogeneous pseudo-embeddings of $H(3,4)$}

The generalized quadrangle $H(3,4)$ is naturally embedded in the projective space $\mathrm{PG}(3,4)$, such that every automorphism of $H(3,4)$ is induced by an automorphism of PG $(3,4)$. This implies that every homogeneous pseudo-embedding of $\mathrm{PG}(3,4)$ induces a homogeneous pseudo-embedding of $H(3,4)$. By [ [8, Theorem 1.4], there are up to isomorphism two homogeneous pseudo-embeddings of $\mathrm{PG}(3,4)$, the universal pseudo-embedding (which has vector dimension 24) and the Hermitian Veronese embedding (which has vector dimension $16)$.

Let $V_{24}^{\prime}$ be an $\mathbb{F}_{2}$-vector space of dimension 24 that meets $V_{24}$ in the subspace $V_{23}$. We denote by $\bar{g}_{34}^{\prime}$ an arbitrary vector of $V_{24}^{\prime} \backslash V_{23}$, and put $V_{16}^{\prime}=\left\langle V_{15}, \bar{g}_{34}^{\prime}\right\rangle$. We define $\bar{g}_{12}^{\prime}:=\bar{g}_{12}+\bar{g}_{34}^{\prime}$ and $\bar{g}_{i j}^{\prime}:=\bar{g}_{i j}$ for all $i, j \in\{1,2,3,4\}$ with $i<j$ and $(1,2) \neq(i, j) \neq(3,4)$.

By [8, Theorem 1.1], the map $\epsilon_{u}: \mathrm{PG}(3,4) \rightarrow \mathrm{PG}\left(V_{24}^{\prime}\right)$ that maps the point $\left(X_{1}, X_{2}, X_{3}\right.$, $\left.X_{4}\right)$ to the point of $\mathrm{PG}\left(V_{24}^{\prime}\right)$ generated by the vector

$$
\begin{aligned}
& \sum_{1} X_{i}^{3} \bar{g}_{i}+\sum_{2}\left(\left(X_{i} X_{j}^{2}+X_{j} X_{i}^{2}\right) \bar{g}_{i j}^{\prime}+\left(\omega X_{i} X_{j}^{2}+\omega^{2} X_{j} X_{i}^{2}\right) \bar{h}_{i j}\right) \\
+ & \sum_{3}\left(\left(X_{i} X_{j} X_{k}+X_{i}^{2} X_{j}^{2} X_{k}^{2}\right) \bar{g}_{i j k}+\left(\omega X_{i} X_{j} X_{k}+\omega^{2} X_{i}^{2} X_{j}^{2} X_{k}^{2}\right) \bar{h}_{i j k}\right)
\end{aligned}
$$

is isomorphic to the universal pseudo-embedding of $\mathrm{PG}(3,4)$. For points $\left(X_{1}, X_{2}, X_{3}, X_{4}\right)$ of $\mathcal{H}$ (satisfying $X_{1} X_{2}^{2}+X_{2} X_{1}^{2}+X_{3} X_{4}^{2}+X_{4} X_{3}^{2}=0$ ), the images of $\epsilon_{u}$ and $\epsilon_{23}$ (as defined in Section 1) coincide. So, $\epsilon_{23}$ determines a homogeneous pseudo-embedding of $H(3,4)$ in a suitable subspace $\Sigma_{23}$ of $\mathrm{PG}\left(V_{23}\right)$.

By [8, Section 3.2], the map $\epsilon_{h}: \mathrm{PG}(3,4) \rightarrow \mathrm{PG}\left(V_{16}^{\prime}\right)$ that maps the point $\left(X_{1}, X_{2}, X_{3}\right.$, $\left.X_{4}\right)$ to the point of $\mathrm{PG}\left(V_{16}^{\prime}\right)$ generated by the vector

$$
\sum_{1} X_{i}^{3} \bar{g}_{i}+\sum_{2}\left(\left(X_{i} X_{j}^{2}+X_{j} X_{i}^{2}\right) \bar{g}_{i j}^{\prime}+\left(\omega X_{i} X_{j}^{2}+\omega^{2} X_{j} X_{i}^{2}\right) \bar{h}_{i j}\right)
$$

is a homogeneous pseudo-embedding (the so-called Hermitian Veronese embedding of $\mathrm{PG}(3,4))$. For points $\left(X_{1}, X_{2}, X_{3}, X_{4}\right)$ of $\mathcal{H}$, the images of $\epsilon_{h}$ and $\epsilon_{15}$ (as defined in Section 1) coincide. So, $\epsilon_{15}$ determines a homogeneous pseudo-embedding of $H(3,4)$ in a suitable subspace $\Sigma_{15}$ of $\mathrm{PG}\left(V_{15}\right)$.

Suppose now that $\mathrm{PG}(3,4)=\mathrm{PG}(V)$ for some vector space $V$ of dimension 4 over $\mathbb{F}_{4}$ and that $\left(\bar{b}_{1}, \bar{b}_{2}, \bar{b}_{3}, \bar{b}_{4}\right)$ is a basis of $V$ such that the point $\left(X_{1}, X_{2}, X_{3}, X_{4}\right)$ of $\mathrm{PG}(3,4)$ coincides with the point $\left\langle\sum_{1} X_{i} \bar{b}_{i}\right\rangle$.

Lemma 4.1. We have $\Sigma_{15}=\operatorname{PG}\left(V_{15}\right)$.

Proof. Considering the points $\left\langle\bar{b}_{i}\right\rangle(i \in\{1,2,3,4\})$ and $\left\langle\bar{b}_{i}+\bar{b}_{j}\right\rangle(i, j \in\{1,2,3,4\}$ with $i<j)$ of $\mathcal{H}$, we see that the points $\left\langle\bar{g}_{i}\right\rangle(i \in\{1,2,3,4\}),\left\langle\bar{h}_{i j}\right\rangle(i, j \in\{1,2,3,4\}$ with $i<j)$ belong to $\Sigma_{15}$. Let $\Sigma^{\prime \prime}$ denote the subspace of $\mathrm{PG}\left(V_{15}\right)$ generated by all these points, and let $\Sigma^{\prime}$ denote the subspace of $\mathrm{PG}\left(V_{15}\right)$ generated by the points $\left\langle\bar{g}_{i j}\right\rangle, i, j \in\{1,2,3,4\}$ with $i<j$ and $(i, j) \neq(3,4)$. Then $\Sigma^{\prime \prime} \subseteq \Sigma_{15}$. As $\operatorname{PG}\left(V_{15}\right)=\left\langle\Sigma^{\prime}, \Sigma^{\prime \prime}\right\rangle$, it thus suffices to show that also $\Sigma^{\prime} \subseteq \Sigma_{15}$. The fact that $\Sigma^{\prime \prime} \subseteq \Sigma_{15}$ implies the following. 
For every point $x$ for which $\epsilon_{15}(x) \notin \Sigma^{\prime \prime}$, we have $\epsilon(x) \in \Sigma_{15}$, where $\epsilon(x)$ is the unique point of $\Sigma^{\prime}$ in the subspace $\left\langle\Sigma^{\prime \prime}, \epsilon_{15}(x)\right\rangle$.

We will now apply this fact to points of $\mathcal{H}$. Note that the above point $\epsilon(x)$ is equal to $\left\langle\sum_{2^{\prime}}\left(X_{i} X_{j}^{2}+X_{j} X_{i}^{2}\right) \bar{g}_{i j}\right\rangle$ which is symmetric in the subindices 1 and 2 , and also in the subindices 3 and 4 .

Considering the points $\left(1, \omega, \omega^{2}, 1\right)$ and $(1, \omega, \omega, 1)$ of $\mathcal{H}$, we find

$$
\begin{gathered}
\left\langle\bar{g}_{12}+\bar{g}_{13}+\bar{g}_{23}+\bar{g}_{24}\right\rangle \in \Sigma_{15}, \\
\left\langle\bar{g}_{12}+\bar{g}_{13}+\bar{g}_{24}\right\rangle \in \Sigma_{15} .
\end{gathered}
$$

From equations (1) and (2), we find $\left\langle\bar{g}_{23}\right\rangle \in \Sigma_{15}$. By applying the symmetries $1 \leftrightarrow 2$ and $3 \leftrightarrow 4$, we then find

$$
\left\langle\bar{g}_{13}\right\rangle,\left\langle\bar{g}_{14}\right\rangle,\left\langle\bar{g}_{23}\right\rangle,\left\langle\bar{g}_{24}\right\rangle \in \Sigma_{15}
$$

From equation (2) and (3), we then find

$$
\left\langle\bar{g}_{12}\right\rangle \in \Sigma_{15}
$$

By (3) and (4), we then know that $\Sigma^{\prime} \subseteq \Sigma_{15}$.

Lemma 4.2. We have $\Sigma_{23}=\operatorname{PG}\left(V_{23}\right)$.

Proof. Considering the points $\left\langle\bar{b}_{i}\right\rangle(i \in\{1,2,3,4\}),\left\langle\bar{b}_{i}+\bar{b}_{j}\right\rangle(i, j \in\{1,2,3,4\}$ with $i<j)$ and $\left\langle\bar{b}_{i}+\bar{b}_{j}+\bar{b}_{k}\right\rangle(i, j, k \in\{1,2,3,4\}$ with $i<j<k)$ of $\mathcal{H}$, we see that the points $\left\langle\bar{g}_{i}\right\rangle(i \in\{1,2,3,4\}),\left\langle\bar{h}_{i j}\right\rangle(i, j \in\{1,2,3,4\}$ with $i<j),\left\langle\bar{h}_{i j k}\right\rangle(i, j, k \in\{1,2,3,4\}$ with $i<j<k)$ belong to $\Sigma_{23}$. Let $\Sigma^{\prime \prime}$ denote the subspace of $\mathrm{PG}\left(V_{23}\right)$ generated by all these points, and let $\Sigma^{\prime}$ denote the subspace of $\mathrm{PG}\left(V_{23}\right)$ generated by the points

$\left\langle\bar{g}_{i j}\right\rangle(i, j \in\{1,2,3,4\}$ with $i<j$ and $(i, j) \neq(3,4)),\left\langle\bar{g}_{i j k}\right\rangle(i, j, k \in\{1,2,3,4\}$ with $i<j<k)$.

Then $\Sigma^{\prime \prime} \subseteq \Sigma_{23}$. As $\mathrm{PG}\left(V_{23}\right)=\left\langle\Sigma^{\prime}, \Sigma^{\prime \prime}\right\rangle$, it thus suffices to show that also $\Sigma^{\prime} \subseteq \Sigma_{23}$. The fact that $\Sigma^{\prime \prime} \subseteq \Sigma_{23}$ implies the following.

For every point $x$ for which $\epsilon_{23}(x) \notin \Sigma^{\prime \prime}$, we have $\epsilon(x) \in \Sigma_{23}$, where $\epsilon(x)$ is the unique point of $\Sigma^{\prime}$ in the subspace $\left\langle\Sigma^{\prime \prime}, \epsilon_{23}(x)\right\rangle$.

We will now apply this fact to several points of $\mathcal{H}$. Note that the above point $\epsilon(x)$ is equal to

$$
\left\langle\sum_{2^{\prime}}\left(X_{i} X_{j}^{2}+X_{j} X_{i}^{2}\right) \bar{g}_{i j}+\sum_{3}\left(X_{i} X_{j} X_{k}+X_{i}^{2} X_{j}^{2} X_{k}^{2}\right) \bar{g}_{i j k}\right\rangle
$$

which is symmetric in the subindices 1 and 2, and also in the subindices 3 and 4 .

Considering the points $(1,1, \omega, 0),(1,1,0, \omega),(1,1, \omega, \omega)$ of $\mathcal{H}$, we see that

$$
\begin{aligned}
& \left\langle\bar{g}_{13}+\bar{g}_{23}+\bar{g}_{123}\right\rangle \in \Sigma_{23}, \\
& \left\langle\bar{g}_{14}+\bar{g}_{24}+\bar{g}_{124}\right\rangle \in \Sigma_{23},
\end{aligned}
$$




$$
\left\langle\bar{g}_{13}+\bar{g}_{14}+\bar{g}_{23}+\bar{g}_{24}+\bar{g}_{123}+\bar{g}_{124}+\bar{g}_{134}+\bar{g}_{234}\right\rangle \in \Sigma_{23} .
$$

From (5), (6) and (7), we deduce

$$
\left\langle\bar{g}_{134}+\bar{g}_{234}\right\rangle \in \Sigma_{23} .
$$

Similarly, by considering the points $(0, \omega, 1,1),(\omega, 0,1,1)$ and $(\omega, \omega, 1,1)$, we find

$$
\left\langle\bar{g}_{123}+\bar{g}_{124}\right\rangle \in \Sigma_{23} \text {. }
$$

Considering the points $\left(1, \omega, \omega^{2}, 1\right)$ and $(1, \omega, \omega, 1)$ of $\mathcal{H}$, we find

$$
\begin{gathered}
\left\langle\bar{g}_{12}+\bar{g}_{13}+\bar{g}_{23}+\bar{g}_{24}+\bar{g}_{124}+\bar{g}_{134}\right\rangle \in \Sigma_{23}, \\
\left\langle\bar{g}_{12}+\bar{g}_{13}+\bar{g}_{24}+\bar{g}_{123}+\bar{g}_{124}+\bar{g}_{134}+\bar{g}_{234}\right\rangle \in \Sigma_{23} .
\end{gathered}
$$

From equations (10) and (11), we find

$$
\left\langle\bar{g}_{23}+\bar{g}_{123}+\bar{g}_{234}\right\rangle \in \Sigma_{23} .
$$

By applying the symmetry $1 \leftrightarrow 2,3 \leftrightarrow 4$ to 12 , we find

$$
\left\langle\bar{g}_{14}+\bar{g}_{124}+\bar{g}_{134}\right\rangle \in \Sigma_{23} .
$$

By (8), (9), (12) and (13), we find

$$
\left\langle\bar{g}_{14}+\bar{g}_{23}\right\rangle \in \Sigma_{23} .
$$

By applying the symmetry $3 \leftrightarrow 4$ to (14), we also find

$$
\left\langle\bar{g}_{13}+\bar{g}_{24}\right\rangle \in \Sigma_{23} .
$$

By (8), (9), (11) and (15), we find

$$
\left\langle\bar{g}_{12}\right\rangle \in \Sigma_{23} .
$$

By considering the point $(\omega, 0,1,1)$ of $\mathcal{H}$, we see that

$$
\left\langle\bar{g}_{13}+\bar{g}_{14}+\bar{g}_{134}\right\rangle \in \Sigma_{23} .
$$

By (6), (13), (15) and (17), we find $\left\langle\bar{g}_{14}\right\rangle \in \Sigma_{23}$. Using the symmetries $1 \leftrightarrow 2,3 \leftrightarrow 4$, we then have

$$
\left\langle\bar{g}_{13}\right\rangle,\left\langle\bar{g}_{14}\right\rangle,\left\langle\bar{g}_{23}\right\rangle,\left\langle\bar{g}_{24}\right\rangle \in \Sigma_{23} .
$$

By (5), (6) and (18), we then have $\left\langle\bar{g}_{123}\right\rangle,\left\langle\bar{g}_{124}\right\rangle \in \Sigma_{23}$. Combining this with (12), (13) and (18), we then find that

$$
\left\langle\bar{g}_{123}\right\rangle,\left\langle\bar{g}_{124}\right\rangle,\left\langle\bar{g}_{134}\right\rangle,\left\langle\bar{g}_{234}\right\rangle \in \Sigma_{23} .
$$

By (16), (18) and (19), we then know that $\Sigma^{\prime} \subseteq \Sigma_{23}$. 
If $x$ and $y$ are two noncollinear points of a generalized quadrangle $\mathcal{Q}$, then $\{x, y\}^{\perp \perp}:=$ $\left(\{x, y\}^{\perp}\right)^{\perp}$ is called a hyperbolic line of $\mathcal{Q}$. If $x$ and $y$ are two points of $H(3,4)$, then $L=\{x, y\}^{\perp}$ and $K=\{x, y\}^{\perp \perp}$ are two hyperbolic lines of $H(3,4)$. Not only do we have $K=L^{\perp}$, but also $L=K^{\perp}$. The hyperbolic lines of $H(3,4)$ are precisely the intersections of size 3 that arise by intersecting $\mathcal{H}$ with the lines of $\mathrm{PG}(3,4)$.

Now, let $L_{12}$ be the hyperbolic line $\{(1,0,0,0),(0,1,0,0),(1,1,0,0)\}$ of $H(3,4)$. Then $L_{34}:=L_{12}^{\perp}=\{(0,0,1,0),(0,0,0,1),(0,0,1,1)\}$ is also a hyperbolic line. A line of $H(3,4)$ contains a (necessarily unique) point of $L_{12}$ if and only if it contains a (necessarily unique) point of $L_{34}$. So, $L_{12} \cup L_{34}$ is an even set, and $H^{*}:=\mathcal{H} \backslash\left(L_{12} \cup L_{34}\right)$ is a pseudo-hyperplane.

Lemma 4.3. The pseudo-hyperplane $H^{*}$ does not arise from the pseudo-embedding $\epsilon_{23}$.

Proof. If $H^{*}$ arises from $\epsilon_{23}$, then $H^{*}$ has an equation of the form

$$
\begin{array}{r}
\sum_{1} a_{i} X_{i}^{3}+\sum_{2^{\prime}} a_{i j}\left(X_{i} X_{j}^{2}+X_{j} X_{i}^{2}\right)+\sum_{2} b_{i j}\left(\omega X_{i} X_{j}^{2}+\omega^{2} X_{j} X_{i}^{2}\right) \\
+\sum_{3} a_{i j k}\left(X_{i} X_{j} X_{k}+X_{i}^{2} X_{j}^{2} X_{k}^{2}\right)+b_{i j k}\left(\omega X_{i} X_{j} X_{k}+\omega^{2} X_{i}^{2} X_{j}^{2} X_{k}^{2}\right)=0,
\end{array}
$$

where

- $a_{i} \in \mathbb{F}_{2}$ for every $i \in\{1,2,3,4\}$;

- $a_{i j} \in \mathbb{F}_{2}$ for all $i, j \in\{1,2,3,4\}$ with $i<j$ and $(i, j) \neq(3,4)$;

- $b_{i j} \in \mathbb{F}_{2}$ for all $i, j \in\{1,2,3,4\}$ with $i<j$;

- $a_{i j k}, b_{i j k} \in \mathbb{F}_{2}$ for all $i, j, k \in\{1,2,3,4\}$ with $i<j<k$.

If $i \in\{1,2\}$ and $j \in\{3,4\}$, then the fact that the points $\left\langle\bar{b}_{i}+\bar{b}_{j}\right\rangle,\left\langle\bar{b}_{i}+\omega \bar{b}_{j}\right\rangle$ and $\left\langle\bar{b}_{i}+\omega^{2} \bar{b}_{j}\right\rangle$ of $H^{*}$ satisfy equation $(*)$ imply that

$$
a_{i}+a_{j}=a_{i j}=b_{i j}=0 .
$$

The latter also implies that

$$
a_{1}=a_{2}=a_{3}=a_{4} .
$$

By (20), 21) and the fact that the points $(1,1,1,0),(1,1, \omega, 0),\left(1,1, \omega^{2}, 0\right),(1,1,0,1)$, $(1,1,0, \omega),\left(1,1,0, \omega^{2}\right),(0,1,1,1),(0, \omega, 1,1),\left(0, \omega^{2}, 1,1\right),(1,0,1,1),(\omega, 0,1,1),\left(\omega^{2}, 0,1,1\right)$ of $H^{*}$ satisfy equation $(*)$, we find

$$
a_{1}=a_{2}=a_{3}=a_{4}=b_{12}=b_{34},
$$

and

$$
a_{i j k}=b_{i j k}=0
$$

for all $i, j, k \in\{1,2,3,4\}$ with $i<j<k$. By (20), (21), (22), (23) and the fact that the points $(1, \omega, 1, \omega)$ and $\left(1, \omega, 1, \omega^{2}\right)$ of $H^{*}$ satisfy equation $(*)$, we then also find that

$$
a_{1}=a_{2}=a_{3}=a_{4}=a_{12}=b_{12}=b_{34}=0 .
$$

So, all coefficients should be zero, which is impossible. 
By Proposition 2.1 and Lemma 4.3, we can enlarge the embedding $\epsilon_{23}$ to a pseudoembedding whose vector dimension is 24. As the pseudo-hyperplane $H^{*} \subseteq \mathcal{H}$ is described by the equation $\left(X_{1}^{3}+X_{2}^{3}+X_{1}^{3} X_{2}^{3}\right)\left(X_{3}^{3}+X_{4}^{3}+X_{3}^{3} X_{4}^{3}\right)=1$, the construction given in Proposition 2.1 tells us that this pseudo-embedding is isomorphic to $\epsilon_{24}$. Proposition 3.1 and Lemma 3.2 then imply the following.

Corollary 4.4. (1) The pseudo-embedding and pseudo-generating ranks of $H(3,4)$ are equal to 24.

(2) The universal pseudo-embedding of $H(3,4)$ is isomorphic to $\epsilon_{24}$.

So far, we have constructed three homogeneous pseudo-embeddings of $H(3,4)$, namely $\epsilon_{15}$, $\epsilon_{23}$ and $\epsilon_{24}$. In Section 6, we classify all homogenous pseudo-embeddings of $H(3,4)$. From that treatment, it follows that there is an additional homogeneous pseudo-embedding of $H(3,4)$. Before we can achieve these goals, we describe in Section 5 a set of generators for the automorphism group of $H(3,4)$ and explain how these act on the even sets of $H(3,4)$.

\section{Generators for the automorphism group of $H(3,4)$}

As before, we put $\mathrm{PG}(3,4)=\mathrm{PG}(V)$, where $V$ is some 4-dimensional $\mathbb{F}_{4}$-vector space, and we suppose the coordinates $\left(X_{1}, X_{2}, X_{3}, X_{4}\right)$ of the points of $\mathrm{PG}(3,4)$ are those with respect to a fixed basis $\left(\bar{b}_{1}, \bar{b}_{2}, \bar{b}_{3}, \bar{b}_{4}\right)$ of $V$. We denote by $h: V \times V \rightarrow \mathbb{F}_{4}$ the Hermitian form described by

$$
h\left(\sum_{i=1}^{4} X_{i} \bar{b}_{i}, \sum_{j=1}^{4} Y_{j} \bar{b}_{j}\right)=X_{1} Y_{2}^{2}+X_{2} Y_{1}^{2}+X_{3} Y_{4}^{2}+X_{4} Y_{3}^{2} .
$$

Note that $\mathcal{H}$ consists of all points $\langle\bar{x}\rangle$ of $\mathrm{PG}(V)$ for which $h(\bar{x}, \bar{x})=0$.

A basis $\left(\bar{e}_{1}, \bar{f}_{1}, \bar{e}_{2}, \bar{f}_{2}\right)$ of $V$ is called a hyperbolic basis of $(V, h)$ if $h\left(\bar{e}_{i}, \bar{e}_{j}\right)=h\left(\bar{f}_{i}, \bar{f}_{j}\right)=0$ and $h\left(\bar{e}_{i}, \bar{f}_{j}\right)=\delta_{i j}$ for all $i, j \in\{1,2\}$. The fixed basis $\left(\bar{b}_{1}, \bar{b}_{2}, \bar{b}_{3}, \bar{b}_{4}\right)$ is thus a hyperbolic basis of $(V, h)$.

Every automorphism $\phi$ of $H(3,4)$ is induced by an automorphism of $\mathrm{PG}(3,4)$ stabilizing $\mathcal{H}$ and so also by a semi-linear transformation $\theta=\theta_{\phi}$ of $V$. For every such $\theta$, there exists an $e_{\theta} \in\{1,2\} \subseteq \mathbb{N}$ such that $h\left(\bar{x}^{\theta}, \bar{y}^{\theta}\right)=h(\bar{x}, \bar{y})^{e_{\theta}}$. We denote by $G$ the subgroup of $\operatorname{Aut}(H(3,4))$ consisting of all $\phi \in \operatorname{Aut}(H(3,4))$ for which $e_{\theta_{\phi}}=1$, i.e. $\theta_{\phi}$ leaves $h$ invariant, or equivalently, $\theta_{\phi}$ maps hyperbolic bases of $(V, h)$ to hyperbolic bases of $(V, h)$.

If $\left(\bar{e}_{1}, \bar{f}_{1}, \bar{e}_{2}, \bar{f}_{2}\right)$ is a hyperbolic basis of $(V, h)$, then obviously

(1) $\left(\bar{e}_{2}, \bar{f}_{2}, \bar{e}_{1}, \bar{f}_{1}\right)$ is also a hyperbolic basis of $(V, h)$;

(2) $\left(\frac{\bar{e}_{1}}{\omega}, \omega^{2} \bar{f}_{1}, \bar{e}_{2}, \bar{f}_{2}\right)$ is also a hyperbolic basis of $(V, h)$;

(3) $\left(\bar{e}_{1}+\bar{e}_{2}, \bar{f}_{1}, \bar{e}_{2}, \bar{f}_{1}+\bar{f}_{2}\right)$ is also a hyperbolic basis of $(V, h)$;

(4) $\left(\bar{e}_{1}, \bar{f}_{1}, \bar{e}_{2}, \bar{f}_{2}+\bar{e}_{2}\right)$ is also a hyperbolic basis of $(V, h)$; 
(5) $\left(\bar{e}_{1}, \bar{f}_{1}, \bar{e}_{2}+\bar{f}_{2}, \bar{e}_{2}\right)$ is also a hyperbolic basis of $(V, h)$.

For every $i \in\{1,2,3,4,5\}$, let $\Omega_{i}$ denote the set of all ordered pairs $\left(B_{1}, B_{2}\right)$ of hyperbolic bases of $(V, h)$ such that $B_{2}$ can be obtained from $B_{1}$ as described in $(i)$ above.

We have thus given five elementary constructions for constructing new hyperbolic bases from given ones. Applying these constructions consecutively, we can obtain other hyperbolic bases. E.g., applying (2) a number of times, we conclude that:

(2') $\left(\frac{\bar{e}_{1}}{\lambda}, \lambda^{2} \bar{f}_{1}, \bar{e}_{2}, \bar{f}_{2}\right)$ is a hyperbolic basis of $(V, h)$ for every $\lambda \in \mathbb{F}_{4}^{*}$.

By applying (2) and (3) a number of times, we can show that also

(3') $\left(\bar{e}_{1}+\lambda \bar{e}_{2}, \bar{f}_{1}, \bar{e}_{2}, \lambda^{2} \bar{f}_{1}+\bar{f}_{2}\right)$ is a hyperbolic basis of $(V, h)$ for every $\lambda \in \mathbb{F}_{4}^{*}$.

Indeed, by (2') we know that $\left(\frac{\bar{e}_{1}}{\lambda}, \lambda^{2} \bar{f}_{1}, \bar{e}_{2}, \bar{f}_{2}\right)$ is a hyperbolic basis of $(V, h)$. By $(3)$, we then know that also $\left(\frac{\bar{e}_{1}}{\lambda}+\bar{e}_{2}, \lambda^{2} \bar{f}_{1}, \bar{e}_{2}, \lambda^{2} \bar{f}_{1}+\bar{f}_{2}\right)$ is a hyperbolic basis of $(V, h)$. Claim (3') then follows by applying (2') once more.

Combining Lemma 2.1 of [7] with (2') and (3'), we then have:

Lemma 5.1. If $B$ and $B^{\prime}$ are two hyperbolic bases of $(V, h)$, then there exist hyperbolic bases $B_{0}, B_{1}, \ldots, B_{k}$ of $(V, h)$ for some $k \in \mathbb{N}$ such that $B_{0}=B, B_{k}=B^{\prime}$ and $\left(B_{i-1}, B_{i}\right) \in$ $\Omega_{1} \cup \Omega_{2} \cup \cdots \cup \Omega_{5}$ for every $i \in\{1,2, \ldots, k\}$.

Now, consider the linear transformations $\theta_{1}, \theta_{2}, \ldots, \theta_{5}$ of $G L(V)$ defined by

- $\left(\bar{b}_{1}, \bar{b}_{2}, \bar{b}_{3}, \bar{b}_{4}\right)^{\theta_{1}}=\left(\bar{b}_{3}, \bar{b}_{4}, \bar{b}_{1}, \bar{b}_{2}\right)$;

- $\left(\bar{b}_{1}, \bar{b}_{2}, \bar{b}_{3}, \bar{b}_{4}\right)^{\theta_{2}}=\left(\frac{\bar{b}_{1}}{\omega}, \omega^{2} \bar{b}_{2}, \bar{b}_{3}, \bar{b}_{4}\right)$;

- $\left(\bar{b}_{1}, \bar{b}_{2}, \bar{b}_{3}, \bar{b}_{4}\right)^{\theta_{3}}=\left(\bar{b}_{1}+\bar{b}_{3}, \bar{b}_{2}, \bar{b}_{3}, \bar{b}_{2}+\bar{b}_{4}\right)$;

- $\left(\bar{b}_{1}, \bar{b}_{2}, \bar{b}_{3}, \bar{b}_{4}\right)^{\theta_{4}}=\left(\bar{b}_{1}, \bar{b}_{2}, \bar{b}_{3}, \bar{b}_{4}+\bar{b}_{3}\right)$;

- $\left(\bar{b}_{1}, \bar{b}_{2}, \bar{b}_{3}, \bar{b}_{4}\right)^{\theta_{5}}=\left(\bar{b}_{1}, \bar{b}_{2}, \bar{b}_{3}+\bar{b}_{4}, \bar{b}_{4}\right)$.

As $\theta_{i}, i \in\{1,2, \ldots, 5\}$, maps every hyperbolic basis of $(V, h)$ to another hyperbolic basis of $(V, h)$, it leaves the Hermitian form $h$ invariant and so defines an automorphism $\phi_{i}$ of $H(3,4)$. Put

$$
\Theta=\left\langle\theta_{1}, \theta_{2}, \theta_{3}, \theta_{4}, \theta_{5}\right\rangle \leq G L(V) .
$$

Then every element of $\Theta$ maps hyperbolic bases of $(V, h)$ to hyperbolic bases of $(V, h)$. The following property is obvious.

Lemma 5.2. Let $\theta \in \Theta$ and $i \in\{1,2,3,4,5\}$. If $\left(\bar{b}_{1}^{\prime}, \bar{b}_{2}^{\prime}, \bar{b}_{3}^{\prime}, \bar{b}_{4}^{\prime}\right)$ is the hyperbolic basis of $(V, h)$ such that $\left(\left(\bar{b}_{1}^{\theta}, \bar{b}_{2}^{\theta}, \bar{b}_{3}^{\theta}, \bar{b}_{4}^{\theta}\right),\left(\bar{b}_{1}^{\prime}, \bar{b}_{2}^{\prime}, \bar{b}_{3}^{\prime}, \bar{b}_{4}^{\prime}\right)\right) \in \Omega_{i}$, then $\theta_{i} \theta=\theta \circ \theta_{i} \in \Theta \operatorname{maps}\left(\bar{b}_{1}, \bar{b}_{2}, \bar{b}_{3}, \bar{b}_{4}\right)$ to $\left(\bar{b}_{1}^{\prime}, \bar{b}_{2}^{\prime}, \bar{b}_{3}^{\prime}, \bar{b}_{4}^{\prime}\right)$.

By Lemmas 5.1 and 5.2 , we then have

Corollary 5.3. $\Theta$ consists of those elements of $G L(V)$ that map hyperbolic bases of $(V, h)$ to hyperbolic bases of $(V, h)$, or equivalently, that leave the form $h$ invariant.

We thus also have 
Corollary 5.4. We have $G=\left\langle\phi_{1}, \phi_{2}, \phi_{3}, \phi_{4}, \phi_{5}\right\rangle$.

If we denote by $\phi_{6}$ the automorphism $\left(X_{1}, X_{2}, X_{3}, X_{4}\right) \mapsto\left(X_{1}^{2}, X_{2}^{2}, X_{3}^{2}, X_{4}^{2}\right)$ of $H(3,4)$, then we have

Corollary 5.5. We have $\operatorname{Aut}(H(3,4))=\left\langle\phi_{1}, \phi_{2}, \phi_{3}, \phi_{4}, \phi_{5}, \phi_{6}\right\rangle$.

We now determine how each of the six generators of $\operatorname{Aut}(H(3,4))$ acts on the even sets of $H(3,4)$, or equivalently, on the corresponding 15 -tuples of the cartesian product $\mathbb{F}_{2}^{6} \times \mathbb{F}_{4}^{9}$ (recall Corollary 1.3. . To that end, we will need to rely on the following three lemmas.

Lemma 5.6. Suppose $E$ is an even set described by an equation $F\left(X_{1}, X_{2}, X_{3}, X_{4}\right)=0$ and let $\phi=\phi_{i}$ with $i \in\{1,2,3,4,5,6\}$. Then the following hold.

- If $i=1$, then $E^{\phi}$ is described by the equation $F\left(X_{3}, X_{4}, X_{1}, X_{2}\right)=0$.

- If $i=2$, then $E^{\phi}$ is described by the equation $F\left(\omega X_{1}, \omega X_{2}, X_{3}, X_{4}\right)=0$.

- If $i=3$, then $E^{\phi}$ is described by the equation $F\left(X_{1}, X_{2}+X_{4}, X_{1}+X_{3}, X_{4}\right)=0$.

- If $i=4$, then $E^{\phi}$ is described by the equation $F\left(X_{1}, X_{2}, X_{3}+X_{4}, X_{4}\right)=0$.

- If $i=5$, then $E^{\phi}$ is described by the equation $F\left(X_{1}, X_{2}, X_{3}, X_{3}+X_{4}\right)=0$.

- If $i=6$, then $E^{\phi}$ is described by the equation $F\left(X_{1}^{2}, X_{2}^{2}, X_{3}^{2}, X_{4}^{2}\right)=0$.

Proof. We give a proof for $i=3$. The treatment for the remaining cases is similar. Suppose $\phi_{3}$ maps to the point $\left(X_{1}, X_{2}, X_{3}, X_{4}\right)$ to the point $\left(Y_{1}, Y_{2}, Y_{3}, Y_{4}\right)$. Then $X_{1}\left(\bar{b}_{1}+\right.$ $\left.\bar{b}_{3}\right)+X_{2} \bar{b}_{2}+X_{3} \bar{b}_{3}+X_{4}\left(\bar{b}_{2}+\bar{b}_{4}\right)=Y_{1} \bar{b}_{1}+Y_{2} \bar{b}_{2}+Y_{3} \bar{b}_{3}+Y_{4} \bar{b}_{4}$. Hence, $\left(Y_{1}, Y_{2}, Y_{3}, Y_{4}\right)=$ $\left(X_{1}, X_{2}+X_{4}, X_{1}+X_{3}, X_{4}\right)$ and $\left(X_{1}, X_{2}, X_{3}, X_{4}\right)=\left(Y_{1}, Y_{2}+Y_{4}, Y_{1}+Y_{3}, Y_{4}\right)$. The latter equality shows that $\left(Y_{1}, Y_{2}, Y_{3}, Y_{4}\right)$ satisfies the equation $F\left(Y_{1}, Y_{2}+Y_{4}, Y_{1}+Y_{3}, Y_{4}\right)=0$.

Lemma 5.7. If $\left(X_{1}, X_{2}, X_{3}, X_{4}\right)$ is a point of $H(3,4)$ and $b \in \mathbb{F}_{4}$, then

$$
b X_{3} X_{4}^{2}+b^{2} X_{4} X_{3}^{2}=\left(b+b^{2}\right)\left(\omega X_{3} X_{4}^{2}+\omega^{2} X_{4} X_{3}^{2}\right)+\left(b \omega^{2}+b^{2} \omega\right)\left(X_{1} X_{2}^{2}+X_{2} X_{1}^{2}\right) .
$$

Proof. We have

$$
\begin{aligned}
b X_{3} X_{4}^{2}+b^{2} X_{4} X_{3}^{2} & =\left(b+b^{2}\right)\left(\omega X_{3} X_{4}^{2}+\omega^{2} X_{4} X_{3}^{2}\right)+\left(b \omega^{2}+b^{2} \omega\right)\left(X_{3} X_{4}^{2}+X_{4} X_{3}^{2}\right) \\
& =\left(b+b^{2}\right)\left(\omega X_{3} X_{4}^{2}+\omega^{2} X_{4} X_{3}^{2}\right)+\left(b \omega^{2}+b^{2} \omega\right)\left(X_{1} X_{2}^{2}+X_{2} X_{1}^{2}\right) .
\end{aligned}
$$

Lemma 5.8. If $\left(X_{1}, X_{2}, X_{3}, X_{4}\right)$ is a point of $H(3,4)$, then

$X_{1} X_{3}^{2} X_{4}^{3}+X_{1}^{2} X_{3} X_{4}^{3}+X_{1}^{3} X_{2} X_{4}^{2}+X_{1}^{3} X_{2}^{2} X_{4}=X_{1} X_{2} X_{4}+\left(X_{1} X_{2} X_{4}\right)^{2}+\left(X_{1} X_{3} X_{4}\right)+\left(X_{1} X_{3} X_{4}\right)^{2}$.

Proof. As $X_{1} X_{2}^{2}+X_{2} X_{1}^{2}+X_{3} X_{4}^{2}+X_{4} X_{3}^{2}=0$, the left hand side is equal to

$$
\begin{aligned}
& X_{1} X_{4}^{2}\left(X_{1}^{2} X_{2}+X_{2}^{2} X_{1}+X_{3} X_{4}^{2}\right)+X_{1}^{2} X_{4}\left(X_{3}^{2} X_{4}+X_{1}^{2} X_{2}+X_{2}^{2} X_{1}\right)+X_{1}^{3} X_{2} X_{4}^{2}+X_{1}^{3} X_{2}^{2} X_{4} \\
& =X_{1}^{2} X_{2}^{2} X_{4}^{2}+X_{1} X_{3} X_{4}^{4}+X_{1}^{2} X_{3}^{2} X_{4}^{2}+X_{1}^{4} X_{2} X_{4}=X_{1} X_{2} X_{4}+\left(X_{1} X_{2} X_{4}\right)^{2}+\left(X_{1} X_{3} X_{4}\right)+\left(X_{1} X_{3} X_{4}\right)^{2} .
\end{aligned}
$$


Lemma 5.9. Let $\phi=\phi_{i} \in\left\{\phi_{1}, \phi_{2}, \ldots, \phi_{6}\right\}$. Suppose $\bar{a}, \bar{a}^{\prime} \in \mathbb{F}_{2}^{6} \times \mathbb{F}_{4}^{9}$ such that $E(\bar{a})^{\phi}=$ $E\left(\bar{a}^{\prime}\right)$. Then $\bar{a}^{\prime}=\left(a_{1}^{\prime}, \ldots, a_{6}^{\prime}, b_{12}^{\prime}, \ldots, b_{234}^{\prime}\right)$ can be obtained from $\bar{a}=\left(a_{1}, \ldots, a_{6}, b_{12}, \ldots, b_{234}\right)$ as described in Tables 1 and 2 .

Proof. We put the left hand side of the expression in Corollary 1.3 equal to $F\left(X_{1}, X_{2}, X_{3}, X_{4}\right)$. By Lemma 5.6, we should then compute $F\left(X_{3}, X_{4}, X_{1}, X_{2}\right), F\left(\omega X_{1}, \omega X_{2}, X_{3}, X_{4}\right), F\left(X_{1}, X_{2}+\right.$ $\left.X_{4}, X_{1}+X_{3}, X_{4}\right), F\left(X_{1}, X_{2}, X_{3}+X_{4}, X_{4}\right), F\left(X_{1}, X_{2}, X_{3}, X_{3}+X_{4}\right)$ and $F\left(X_{1}^{2}, X_{2}^{2}, X_{3}^{2}, X_{4}^{2}\right)$. Expanding each of the latter polynomials, we obtain a sum of terms of the form:

- $X_{i}^{3}$ with $i \in\{1,2,3,4\}$;

- $\lambda X_{i} X_{j}^{2}+\lambda^{2} X_{j} X_{i}^{2}$ with $\lambda \in\{1, \omega\}$ and $i, j \in\{1,2,3,4\}$ with $i<j$;

- $\lambda X_{i} X_{j} X_{k}+\lambda^{2} X_{i}^{2} X_{j}^{2} X_{k}^{2}$ with $\lambda \in\{1, \omega\}$ and $i, j, k \in\{1,2,3,4\}$ with $i<j<k$;

- $\left(X_{1}^{3}+X_{2}^{3}+X_{1}^{3} X_{2}^{3}\right)\left(X_{3}^{3}+X_{4}^{3}+X_{3}^{3} X_{4}^{3}\right)+1$

- $X_{1} X_{3}^{2} X_{4}^{3}+X_{1}^{2} X_{3} X_{4}^{3}+X_{1}^{3} X_{2} X_{4}^{2}+X_{1}^{3} X_{2}^{2} X_{4}$.

Collecting terms in the expansion, we get a linear combination of the required shape (as in Corollary 1.3), with exception of the terms of the form $X_{1} X_{3}^{2} X_{4}^{3}+X_{1}^{2} X_{3} X_{4}^{3}+X_{1}^{3} X_{2} X_{4}^{2}+$ $X_{1}^{3} X_{2}^{2} X_{4}$ and $b X_{3} X_{4}^{2}+b^{2} X_{4} X_{3}^{2}$ for some $b \in \mathbb{F}_{4}$. By Lemmas 5.7 and 5.8, also these terms can be written in the right shape.

E.g., for $\phi=\phi_{3}$, the factor for $a_{6}$ in the expression $F\left(X_{1}, X_{2}+X_{4}, X_{1}+X_{3}, X_{4}\right)$ is equal to

$$
\begin{gathered}
\left(\left(X_{1}^{3}+X_{2}^{3}+X_{1}^{3} X_{2}^{3}\right)\left(X_{3}^{3}+X_{4}^{3}+X_{3}^{3} X_{4}^{3}\right)+1\right)+X_{1}^{3}+X_{4}^{3}+X_{2} X_{4}^{2}+X_{4} X_{2}^{2} \\
+X_{1} X_{3}^{2}+X_{3} X_{1}^{2}+X_{1} X_{3}^{2} X_{4}^{3}+X_{1}^{2} X_{3} X_{4}^{3}+X_{1}^{3} X_{2} X_{4}^{2}+X_{1}^{3} X_{2}^{2} X_{4} .
\end{gathered}
$$

\section{The homogeneous pseudo-embeddings of $H(3,4)$}

In this section, we classify all homogeneous pseudo-embeddings of the generalized quadrangle $H(3,4)$. Our classification relies on the following results from [8, 10].

Proposition 6.1 ([10, Corollary 2.7]). Let $\mathcal{S}=(\mathcal{P}, \mathcal{L}, \mathrm{I})$ be a point-line geometry with the property that the number of points on each line is finite and at least three, and let $G$ be a group of automorphisms of $\mathcal{S}$.

If $\epsilon: \mathcal{S} \rightarrow \Sigma$ is a $G$-homogeneous pseudo-embedding of $\mathcal{S}$, then the set $\mathcal{H}=\mathcal{H}_{\epsilon}$ satisfies the following properties:

(a) $\mathcal{H}$ is a union of $G$-orbits of pseudo-hyperplanes;

(b) if $H_{1}$ and $H_{2}$ are two distinct elements of $\mathcal{H}$, then the complement of the symmetric difference of $H_{1}$ and $H_{2}$ also belongs to $\mathcal{H}$;

(c) if $L$ is a line of $\mathcal{S}$ containing an odd number of points, then for every point $x$ of $L$ there exists a pseudo-hyperplane of $\mathcal{H}$ that only has the point $x$ in common with $L$;

(d) if $L$ is a line of $\mathcal{S}$ containing an even number of points, then for any two distinct points $x_{1}$ and $x_{2}$ of $L$, there exists a pseudo-hyperplane of $\mathcal{H}$ having only the points $x_{1}$ and $x_{2}$ in common with $L$; 


\begin{tabular}{|c||c|c|c|}
\hline$i$ & 1 & 2 & 3 \\
\hline \hline$a_{1}^{\prime}$ & $a_{3}$ & $a_{1}$ & $a_{1}+a_{3}+a_{6}+b_{13}+b_{13}^{2}$ \\
\hline$a_{2}^{\prime}$ & $a_{4}$ & $a_{2}$ & $a_{2}$ \\
\hline$a_{3}^{\prime}$ & $a_{1}$ & $a_{3}$ & $a_{3}$ \\
\hline$a_{4}^{\prime}$ & $a_{2}$ & $a_{4}$ & $a_{4}+a_{2}+a_{6}+b_{24}+b_{24}^{2}$ \\
\hline$a_{5}^{\prime}$ & $b_{12}+b_{12}^{2}$ & $a_{5}$ & $a_{5}+b_{23}+b_{23}^{2}+b_{234}+b_{234}^{2}$ \\
\hline$a_{6}^{\prime}$ & $a_{6}$ & $a_{6}$ & $a_{6}$ \\
\hline$b_{12}^{\prime}$ & $\omega a_{5}+\omega^{2} b_{12}+\omega b_{12}^{2}$ & $b_{12}$ & $b_{12}+b_{123}^{2}+\omega\left(b_{23}+b_{23}^{2}+\omega^{2} b_{234}+\omega b_{234}^{2}\right.$ \\
\hline$b_{13}^{\prime}$ & $b_{13}^{2}$ & $\omega b_{13}$ & $b_{13}+a_{3}+a_{6}$ \\
\hline$b_{14}^{\prime}$ & $b_{23}^{2}$ & $\omega b_{14}$ & $b_{14}+\omega a_{5}+b_{12}+b_{23}^{2}+b_{123}^{2}+b_{124}+b_{134}^{2}+b_{234}$ \\
\hline$b_{23}^{\prime}$ & $b_{14}^{2}$ & $\omega b_{23}$ & $b_{23}$ \\
\hline$b_{24}^{\prime}$ & $b_{24}^{2}$ & $\omega b_{24}$ & $b_{24}+a_{2}+a_{6}$ \\
\hline$b_{123}^{\prime}$ & $b_{134}$ & $\omega^{2} b_{123}$ & $b_{123}$ \\
\hline$b_{124}^{\prime}$ & $b_{234}$ & $\omega^{2} b_{124}$ & $b_{124}+a_{6}+b_{234}$ \\
\hline$b_{134}^{\prime}$ & $b_{123}$ & $\omega b_{134}$ & $b_{6}+b_{123}$ \\
\hline$b_{234}^{\prime}$ & $b_{124}$ & $\omega b_{234}$ & \\
\hline & & & $b_{234}$ \\
\hline
\end{tabular}

Table 1: The action of $\phi_{1}, \phi_{2}$ and $\phi_{3}$ on the even sets

\begin{tabular}{|c||c|c|c|}
\hline$i$ & 4 & 5 & 6 \\
\hline \hline$a_{1}^{\prime}$ & $a_{1}$ & $a_{1}$ & $a_{1}$ \\
\hline$a_{2}^{\prime}$ & $a_{2}$ & $a_{2}$ & $a_{2}$ \\
\hline$a_{3}^{\prime}$ & $a_{3}$ & $a_{3}+a_{4}+a_{5}$ & $a_{3}$ \\
\hline$a_{4}^{\prime}$ & $a_{4}+a_{3}+a_{5}$ & $a_{4}$ & $a_{4}$ \\
\hline$a_{5}^{\prime}$ & $a_{5}$ & $a_{5}$ & $a_{5}$ \\
\hline$a_{6}^{\prime}$ & $a_{6}$ & $a_{6}$ & $a_{6}$ \\
\hline$b_{12}^{\prime}$ & $b_{12}+a_{3}$ & $b_{12}+a_{4}$ & $b_{12}^{2}+a_{5}$ \\
\hline$b_{13}^{\prime}$ & $b_{13}$ & $b_{13}+b_{14}+b_{134}$ & $b_{13}^{2}$ \\
\hline$b_{14}^{\prime}$ & $b_{14}+b_{13}+b_{134}$ & $b_{14}$ & $b_{14}^{2}$ \\
\hline$b_{23}^{\prime}$ & $b_{23}$ & $b_{23}+b_{24}+b_{234}$ & $b_{23}^{2}$ \\
\hline$b_{24}^{\prime}$ & $b_{24}+b_{23}+b_{234}$ & $b_{24}$ & $b_{24}^{2}$ \\
\hline$b_{123}^{\prime}$ & $b_{123}$ & $b_{123}+b_{124}$ & $b_{123}^{2}$ \\
\hline$b_{124}^{\prime}$ & $b_{124}+b_{123}$ & $b_{124}$ & $b_{124}^{2}$ \\
\hline$b_{134}^{\prime}$ & $b_{134}$ & $b_{134}$ & $b_{134}^{2}$ \\
\hline$b_{234}^{\prime}$ & $b_{234}$ & $b_{234}$ & $b_{234}^{2}$ \\
\hline
\end{tabular}

Table 2: The action of $\phi_{4}, \phi_{5}$ and $\phi_{6}$ on the even sets 
(e) for every point $x$ of $\mathcal{S}$, there exists a pseudo-hyperplane of $\mathcal{H}$ not containing $x$.

Conversely, if $\mathcal{H}$ is a finite set of pseudo-hyperplanes of $\mathcal{S}$ satisfying the conditions $(a)$, $(b),(c),(d)$ and $(e)$ above, then there exists a pseudo-embedding $\epsilon$ of $\mathcal{S}$ such that $\mathcal{H}=\mathcal{H}_{\epsilon}$. This pseudo-embedding $\epsilon$ is moreover unique, up to isomorphism, and G-homogeneous.

Proposition 6.2 ([8, Theorem 3.1]). Let $\mathcal{S}=(\mathcal{P}, \mathcal{L}, \mathrm{I})$ be a point-line geometry with the property that the number of points on each line is finite and at least three. Let $V_{1}$ and $V_{2}$ be two vector spaces over $\mathbb{F}_{2}$. For every $i \in\{1,2\}$, let $\epsilon_{i}$ be a map from the point set $\mathcal{P}$ of $\mathcal{S}$ to the point set of $\mathrm{PG}\left(V_{i}\right)$ and let $\mathcal{H}_{i}$ be the set of all sets of the form $\epsilon_{i}^{-1}\left(\epsilon_{i}(\mathcal{P}) \cap \Pi\right)$, where $\Pi$ is some hyperplane of $\mathrm{PG}\left(V_{i}\right)$. If $\epsilon_{1}$ is a pseudo-embedding of $\mathcal{S}$ and $\mathcal{H}_{1}=\mathcal{H}_{2}$, then also $\epsilon_{2}$ is a pseudo-embedding of $\mathcal{S}$. Moreover, $\epsilon_{2}$ is isomorphic to $\epsilon_{1}$.

In order to use Proposition 6.1 in the case of $H(3,4)$, we first translate the conditions of Proposition 6.1 to conditions that must be satisfied by the corresponding even sets, and subsequently to conditions that must be satisfied by the corresponding elements of $\mathbb{F}_{2}^{6} \times \mathbb{F}_{4}^{9}$.

If $\epsilon: H(3,4) \rightarrow \Sigma$ is a pseudo-embedding of $H(3,4)$, then we denote by $\mathcal{E}_{\epsilon}$ the complements of the elements of $\mathcal{H}_{\epsilon}$. Every element of $\mathcal{E}_{\epsilon}$ is an even set. We put $\mathcal{A}_{\epsilon}:=$ $\left\{\bar{a}_{E} \mid E \in \mathcal{E}_{\epsilon}\right\} \cup\{(0,0, \ldots, 0)\}$, where $\bar{a}_{E}$ with $E \in \mathcal{E}_{\epsilon}$ denotes the unique element $\bar{a} \in$ $\mathbb{F}_{2}^{6} \times \mathbb{F}_{4}^{9}$ for which $E(\bar{a})=E$.

Lemma 6.3. If $\epsilon$ is a homogeneous pseudo-embedding of $H(3,4)$, then the set $\mathcal{E}:=\mathcal{E}_{\epsilon}$ satisfies the following properties:

(a) $\mathcal{E}$ can be written as a union of isomorphism classes of even sets;

(b) the symmetric difference of two distinct even sets of $\mathcal{E}$ is again an even set of $\mathcal{E}$;

(c) $\mathcal{E} \neq \emptyset$

Conversely, if $\mathcal{E}$ is a set of nontrivial even sets of $H(3,4)$ satisfying the properties $(a)$, $(b)$ and $(c)$ above, then there exists a pseudo-embedding $\epsilon$ of $\mathcal{S}$ such that $\mathcal{E}=\mathcal{E}_{\epsilon}$. This pseudo-embedding $\epsilon$ is moreover unique, up to isomorphism, and homogeneous.

Proof. The fact that the conditions (a), (b) and (c) are satisfied by any homogeneous pseudo-embedding of $H(3,4)$ is a consequence of conditions (a), (b) and (c) of Proposition 6.1 .

Conversely, suppose that $\mathcal{E}$ is a set of nontrivial even sets of $H(3,4)$ satisfying the conditions (a), (b) and (c). Let $\mathcal{G}$ denote the full group of automorphisms of $H(3,4)$ and let $\mathcal{H}$ denote the set of all complements of the elements of $\mathcal{E}$. Then $\mathcal{H} \neq \emptyset$ by condition (c). The set $\mathcal{H}$ satisfies the conditions (a) and (b) of Proposition 6.1 as the conditions (a) and (b) of the present lemma are satisfied. Also Property (d) of Proposition 6.1 is satisfied as there are no lines containing an even number of points. Property (e) of Proposition 6.1 is satisfied as $\mathcal{H} \neq \emptyset$ and $\mathcal{G}$ is point-transitive. In view of Proposition 6.1, we thus see 
that the lemma will be valid if we can also show that Property (c) of that proposition is satisfied. For that purpose, we take an arbitrary flag $(x, L)$ of $H(3,4)$.

We show that there exists an element of $\mathcal{E}$ intersecting some line of $H(3,4)$ in four points. Suppose that this is not the case. Then there exists an element $E$ of $\mathcal{E}$ intersecting a certain line $M$ in exactly two points $x_{1}$ and $x_{2}$. Let $x_{3}$ and $x_{4}$ be two other points on the line $M$. As $\mathcal{G}$ acts transitively on the pairs of distinct collinear points, there exists an automorphism $\phi$ of $H(3,4)$ such that $E^{\phi} \cap M=\left\{x_{3}, x_{4}\right\}$. But then $E \Delta E^{\phi}$ intersects $M$ in $\left\{x_{1}, x_{2}, x_{3}, x_{4}\right\}$. This is a contradiction as $E \Delta E^{\phi} \in \mathcal{E}$ by properties (a) and (b).

So, there exists an element of $\mathcal{E}$ intersecting some line of $H(3,4)$ in four points. As $\mathcal{G}$ is flag-transitive, we then know that there also exists an element of $\mathcal{E}$ intersecting $L$ in $L \backslash\{x\}$. The complement of this even set belongs to $\mathcal{H}$ and intersects $L$ in $\{x\}$.

The action of $\phi_{i}, i \in\{1,2, \ldots, 6\}$, on the even sets defines a linear transformation $\sigma_{i}$ on the $\mathbb{F}_{2}$ vector space $\mathbb{F}_{2}^{6} \times \mathbb{F}_{4}^{9}$. The description of these linear transformations can be found in Tables 1 and 2 ,

Lemma 6.4. If $\epsilon$ is a homogeneous pseudo-embedding of $H(3,4)$, then the set $\mathcal{A}_{\epsilon}$ is a subspace of dimension at least 1 of the $\mathbb{F}_{2}$-vector space $\mathbb{F}_{2}^{6} \times \mathbb{F}_{4}^{9}$ that is closed under the linear maps $\sigma_{1}, \sigma_{2}, \ldots, \sigma_{6}$. Conversely, every subspace $\mathcal{A}$ of dimension at least 1 of the $\mathbb{F}_{2}$-vector space $\mathbb{F}_{2}^{6} \times \mathbb{F}_{4}^{9}$ that is closed under the maps $\sigma_{1}, \sigma_{2}, \ldots, \sigma_{6}$ is of the form $\mathcal{A}_{\epsilon}$ for some homogeneous pseudo-embedding $\epsilon$ of $H(3,4)$. This pseudo-embedding $\epsilon$ is moreover unique, up to isomorphism.

Proof. Suppose $\mathcal{E}$ is a set of nontrivial even sets and $\mathcal{A}:=\left\{\bar{a}_{E} \mid E \in \mathcal{E}\right\} \cup\{(0,0, \ldots, 0)\}$. The condition (a) of Lemma 6.3 is equivalent with the claim that $\mathcal{A}$ is closed under the linear maps $\sigma_{1}, \sigma_{2}, \ldots, \sigma_{6}$, the condition (b) of Lemma 6.3 is equivalent with the claim that $\mathcal{A}$ is a subspace of the $\mathbb{F}_{2}$-vector space $\mathbb{F}_{2}^{6} \times \mathbb{F}_{4}^{9}$, and the condition (c) of Lemma 6.3 is equivalent with the claim that the dimension of the subspace $\mathcal{A}$ is at least one. Taking these facts into account, we see that Lemma 6.4 is now a consequence of Lemma 6.3.

We now use Lemma 6.4 to classify all homogeneous pseudo-embeddings of $H(3,4)$. We define the following subspaces of $\mathbb{F}_{2}^{6} \times \mathbb{F}_{4}^{9}$ :

- $W_{24}=\mathbb{F}_{2}^{6} \times \mathbb{F}_{4}^{9}$

- $W_{23}$ consists of those $\bar{a} \in \mathbb{F}_{2}^{6} \times \mathbb{F}_{4}^{9}$ for which $a_{6}=0$;

- $W_{15}$ consists of those $\bar{a} \in \mathbb{F}_{2}^{6} \times \mathbb{F}_{4}^{9}$ for which $a_{6}=0$ and $b_{123}=b_{124}=b_{134}=b_{234}=0$.

- $W_{14}$ consists of those $\bar{a} \in \mathbb{F}_{2}^{6} \times \mathbb{F}_{4}^{9}$ for which $a_{6}=0, b_{123}=b_{124}=b_{134}=b_{234}=0$ and $a_{5}=b_{12}+b_{12}^{2}$. Putting $b_{12}=b_{12}^{\prime}+b_{12}^{\prime \prime} \omega$, with $b_{12}^{\prime}, b_{12}^{\prime \prime} \in \mathbb{F}_{2}$, the latter condition is equivalent with $a_{5}=b_{12}^{\prime \prime}$.

Using the explicit actions of $\sigma_{1}, \sigma_{2}, \ldots, \sigma_{6}$, we see that each of these subspaces is stabilized by these maps. By Lemma 6.4, there are associated pseudo-embeddings $\epsilon_{24}^{\prime}, \epsilon_{23}^{\prime}, \epsilon_{15}^{\prime}$ and $\epsilon_{14}^{\prime}$. By Proposition 6.2, these pseudo-embeddings are respectively isomorphic to $\epsilon_{24}, \epsilon_{23}$, $\epsilon_{15}$ and $\epsilon_{14}$. 
In order to complete the proof of Theorem 1.2 , it suffices to prove that $W_{24}, W_{23}$, $W_{15}$ and $W_{14}$ are the only subspaces of dimension at least one of $\mathbb{F}_{2}^{6} \times \mathbb{F}_{4}^{9}$ stabilized by $\sigma_{1}, \sigma_{2}, \ldots, \sigma_{6}$.

In the sequel, let $W$ be a subspace of dimension at least one of $\mathbb{F}_{2}^{6} \times \mathbb{F}_{4}^{9}$ that is stabilized by $\sigma_{1}, \sigma_{2}, \ldots, \sigma_{6}$. For every $i \in\{1,2, \ldots, 6\}$, let $\Omega_{i}$ denote the set of all vectors of $\mathbb{F}_{2}^{6} \times \mathbb{F}_{4}^{9}$ all whose coordinates are zero, except possibly for the $a_{i}$-coordinates. For every $I \in$ $\{12,13, \ldots, 234\}$, let $\Omega_{I}$ denote the set of all vectors of $\mathbb{F}_{2}^{6} \times \mathbb{F}_{4}^{9}$ all whose coordinates are zero, except possibly for the $b_{I}$-coordinates.

Lemma 6.5. If $\bar{a}=\left(a_{1}, \ldots, a_{6}, b_{12}, \ldots, b_{24}, b_{123}, \ldots, b_{234}\right)$ belongs to $W$, then also $(0,0,0$, $\left.0,0,0,0, b_{13}, b_{14}, b_{23}, b_{24}, b_{123}, b_{124}, b_{134}, b_{234}\right)$ belongs to $W$.

Proof. The latter vector is precisely $\bar{a}^{\sigma_{2} \sigma_{2}}+\bar{a}^{\sigma_{2}}$.

Lemma 6.6. If $W$ contains a nonzero vector all whose coordinates are zero with exception of the $b_{14}$-coordinate, then $W$ contains $W_{14}$.

Proof. Since $W^{\sigma_{2}}=W$, we then see that $\Omega_{14} \subseteq W$. Since $\Omega_{14} \subseteq W$ and $W^{\sigma_{1}}=W$, we have $\Omega_{23} \subseteq W$. Since $\Omega_{23} \subseteq W$ and $\bar{a}+\bar{a}^{\sigma_{4}} \in W$ for all $\bar{a} \in W$, we see that $\Omega_{24} \subseteq W$. Since $\Omega_{14} \subseteq W$ and $\bar{a}+\bar{a}^{\sigma_{5}} \in W$ for all $\bar{a} \in W$, we see that $\Omega_{13} \subseteq W$. Since $\left\langle\Omega_{13}, \Omega_{24}\right\rangle \subseteq W$ and $\bar{a}+\bar{a}^{\sigma_{3}} \in W$ for all $\bar{a} \in W$, we see that $\left\langle\Omega_{1}, \Omega_{4}\right\rangle \subseteq W$. Since $\left\langle\Omega_{1}, \Omega_{4}\right\rangle \subseteq W$ and $W^{\sigma_{1}}=W$, we have $\left\langle\Omega_{2}, \Omega_{3}\right\rangle \subseteq W$.

Since $\left\langle\Omega_{1}, \Omega_{2}, \Omega_{3}, \Omega_{4}, \Omega_{13}, \Omega_{14}, \Omega_{23}, \Omega_{24}\right\rangle \subseteq W$ and $\bar{a}+\bar{a}^{\sigma_{4}} \in W$ for all $\bar{a} \in W$, we see that $W$ contains all vectors of $\left\langle\Omega_{5}, \Omega_{12}\right\rangle$ of the form $\left(0,0,0,0,0,0, b_{12}, 0, \ldots, 0\right)$ with $b_{12} \in \mathbb{F}_{2}$.

Since $\left\langle\Omega_{1}, \Omega_{2}, \Omega_{3}, \Omega_{4}, \Omega_{13}, \Omega_{14}, \Omega_{23}, \Omega_{24}\right\rangle \subseteq W$ and $\bar{a}+\bar{a}^{\sigma_{3}} \in W$ for all $\bar{a} \in W$, we see that $W$ contains all vectors of $\left\langle\Omega_{5}, \Omega_{12}\right\rangle$ of the form $\left(0,0,0,0, a_{5}, 0, a_{5} \omega, 0, \ldots, 0\right)$ with $a_{5} \in \mathbb{F}_{2}$.

All together, we thus see that $W$ contains the subspace $W_{14}$.

Lemma 6.7. If $b_{123}=0$ for all vectors of $W$, then $W$ is equal to either $W_{14}$ or $W_{15}$.

Proof. We first prove that $W \subseteq W_{15}$. The fact that certain coordinates are equal to 0 for all vectors of $W$ implies that other coordinates are as well 0 for all vectors of $W$ :

- as $b_{123}=0$ and $W^{\sigma_{1}}=W^{\sigma_{5}}=W$, we have $b_{134}=b_{124}=0$;

- as $b_{123}=b_{134}=0$ and $W^{\sigma_{3}}=W$, we have $a_{6}=0$;

- as $b_{124}=0$ and $W^{\sigma_{1}}=W$, we have $b_{234}=0$.

So, we indeed have $W \subseteq W_{15}$.

We show that it is not possible that $b_{23}=0$ for all vectors of $W \subseteq W_{15}$. Suppose to the contrary that $b_{23}=0$ for all vectors of $W$. The fact that $W^{\sigma_{1}}=W^{\sigma_{5}}=W$ then implies that also $b_{14}=b_{24}=0$ for all vectors of $W$. Since $W^{\sigma_{4}}=W$ and $b_{14}=0$ for all vectors of $W$, we then know that $b_{13}=0$ for all vectors of $W$. As $W^{\sigma_{3}}=W$ and $b_{13}=b_{24}=0$ for all vectors of $W$, we know that $a_{2}=a_{3}=0$ for all vectors of $W$. This in combination with $W^{\sigma_{1}}=W$ implies that $a_{1}=a_{4}=0$ for all vectors of $W$. Since $W^{\sigma_{4}}=W$ and $a_{3}=a_{4}=0$ for all vectors of $W$, we also know that $a_{5}=0$ for all vectors of $W$. Since $W^{\sigma_{3}}=W$ and 
$b_{14}=a_{5}=b_{23}=b_{123}=b_{124}=b_{134}=b_{234}=0$ for all vectors of $W$, we also know that $b_{12}=0$ for all vectors of $W$. So, $W=0$, an obvious contradiction.

So, we know that $W$ contains a vector whose $b_{23}$-coordinate is distinct from 0 . In combination with Lemma 6.5 this implies that $W \subseteq W_{15}$ contains a vector $\bar{a}=(0,0,0,0,0,0,0$, $\left.b_{13}, b_{14}, b_{23}, b_{24}, 0,0,0,0\right)$ with $b_{23} \neq 0$. Lemma 6.5 and the fact that $\bar{a}^{\sigma_{3}}+\bar{a} \in W$ then implies that $\left(0,0,0,0,0,0,0,0, b_{23}^{2}, 0,0,0,0,0,0\right) \in W$. By Lemma 6.6, we know that $W_{14} \subseteq W$. As $W \subseteq W_{15}$ and $W_{14}$ is a hyperplane of $W_{15}$, we then know that $W$ is either $W_{14}$ or $W_{15}$.

Lemma 6.8. If $W$ contains a vector $\bar{a}$ whose $b_{123}$-coordinate is distinct from 0 , then $W$ is equal to $W_{23}$ or $W_{24}$.

Proof. As $W_{23}$ is a hyperplane of $W_{24}$, it suffices to prove that $W_{23} \subseteq W$.

We first show that $W$ contains a nonzero vector all whose coordinates are 0 with exception of the $b_{14}$-coordinate. By Lemma 6.5, $W$ contains the vector

$$
\bar{a}_{1}=\left(0,0,0,0,0,0,0, b_{13}, b_{14}, b_{23}, b_{24}, b_{123}, b_{124}, b_{134}, b_{234}\right)
$$

and hence also the vector

$$
\bar{a}_{2}=\bar{a}_{1}+\bar{a}_{1}^{\sigma_{4}}=\left(0,0,0,0,0,0,0,0, b_{13}+b_{134}, 0, b_{23}+b_{234}, 0, b_{123}, 0,0\right) .
$$

Thus $W$ also contains the vector

$$
\bar{a}_{2}+\bar{a}_{2}^{\sigma_{3}}=\left(0,0,0, b_{23}+b_{23}^{2}+b_{234}+b_{234}^{2}, 0,0,0,0, b_{123}, 0,0,0,0,0,0\right)
$$

and hence also the vector $\left(0,0,0,0,0,0,0,0, b_{123}, 0,0,0,0,0,0\right)$ by Lemma 6.5. By Lemma 6.6, we thus already know that $W_{14} \subseteq W$. By Lemma 6.5, the fact that $W_{14} \subseteq W$ and the fact that $\bar{a}^{\sigma_{4}}+\bar{a} \in W$ for all $\bar{a} \in W$, we then know that $W$ contains a nonzero vector of $\Omega_{124}$. As $W^{\sigma_{2}}=W$, we then know that $\Omega_{124} \subseteq W$. As $\Omega_{124} \subseteq W$ and $W^{\sigma_{1}}=W$, we then know that $\Omega_{234} \subseteq W$. Since $\Omega_{124} \subseteq W$ and $\bar{a}+\bar{a}^{\sigma_{5}} \in W$ for all $\bar{a} \in W$, we have $\Omega_{123} \subseteq W$. As $\Omega_{123} \subseteq W$ and $W^{\sigma_{1}}=W$, we have $\Omega_{134} \subseteq W$. Since $\left\langle\Omega_{1}, \Omega_{2}, \Omega_{3}, \Omega_{4}, \Omega_{13}, \Omega_{14}, \Omega_{23}, \Omega_{24}, \Omega_{123}, \Omega_{124}, \Omega_{134}, \Omega_{234}\right\rangle \subseteq W$ and $W^{\sigma_{3}}=W$, we have $\left\langle\Omega_{5}, \Omega_{12}\right\rangle \subseteq W$. All together, we thus know that $W_{23} \subseteq W$.

By Lemmas 6.7 and 6.8, we have:

Corollary 6.9. $W$ is equal to either $W_{14}, W_{15}, W_{23}$ and $W_{24}$.

\section{The hyperovals of $H(3,4)$}

Every hyperoval of $H(3,4)$ is an example of an even set, i.e. a set of the form $E(\bar{a})$, where $\bar{a} \in \mathbb{F}_{2}^{6} \times \mathbb{F}_{4}^{9}$. With the aid of the Computer Algebra System GAP [19], we have determined for which of the $2^{24}$ elements $\bar{a} \in \mathbb{F}_{2}^{6} \times \mathbb{F}_{4}^{9}$, the even set $E(\bar{a})$ is a hyperoval, see [11]. It turns out that this is the case for 70648 such tuples. 


\begin{tabular}{|c||c|c|c|c|c|}
\hline$H$ & $v$ & $N$ & Stabilizer $S_{1}$ & $O_{1}$ & $O_{2}$ \\
\hline \hline$H_{6}$ & 6 & 120 & $S_{3} \times\left(\left(S_{3} \times S_{3}\right): C_{2}\right)$ & 1 & 2 \\
\hline$H_{8}$ & 8 & 540 & $C_{2} \times C_{2} \times S_{4}$ & 1 & 4 \\
\hline$H_{10 a}$ & 10 & 216 & $C_{2} \times S_{5}$ & 1 & 2 \\
\hline$H_{10 b}$ & 10 & 2160 & $D_{24}$ & 2 & 5 \\
\hline$H_{10 c}$ & 10 & 2592 & $D_{20}$ & 1 & 5 \\
\hline$H_{12 a}$ & 12 & 120 & $\left(\left(S_{3} \times S_{3}\right): C_{2}\right) \times S_{3}$ & 1 & 2 \\
\hline$H_{12 b}$ & 12 & 2160 & $C_{2} \times C_{2} \times S_{3}$ & 1 & 7 \\
\hline$H_{12 c}$ & 12 & 2880 & $D_{18}$ & 2 & 5 \\
\hline$H_{12 d}$ & 12 & 6480 & $D_{8}$ & 3 & 8 \\
\hline$H_{14 a}$ & 14 & 4320 & $D_{12}$ & 4 & 6 \\
\hline$H_{14 b}$ & 14 & 4320 & $D_{12}$ & 3 & 7 \\
\hline$H_{14 c}$ & 14 & 4320 & $D_{12}$ & 3 & 7 \\
\hline$H_{14 d}$ & 14 & 6480 & $D_{8}$ & 3 & 8 \\
\hline$H_{16 a}$ & 16 & 540 & $G L(2,3): C_{2}$ & 1 & 3 \\
\hline$H_{16 b}$ & 16 & 2160 & $S_{4}$ & 2 & 5 \\
\hline$H_{16 c}$ & 16 & 6480 & $C_{2} \times C_{2} \times C_{2}$ & 3 & 9 \\
\hline$H_{16 d}$ & 16 & 8640 & $S_{3}$ & 4 & 8 \\
\hline$H_{18 a}$ & 18 & 40 & $\left.\left(\left(\left(C_{3} \times\left(\left(C_{3} \times C_{3}\right): C_{2}\right)\right): C_{2}\right): C_{3}\right): C_{2}\right): C_{2}$ & 1 & 1 \\
\hline$H_{18 b}$ & 18 & 240 & $\left(\left(\left(\left(C_{3} \times C_{3}\right): C_{3}\right): C_{2}\right): C_{2}\right): C_{2}$ & 1 & 2 \\
\hline$H_{18 c}$ & 18 & 1440 & $S_{3} \times S_{3}$ & 3 & 3 \\
\hline$H_{18 d}$ & 18 & 1440 & $S_{3} \times S_{3}$ & 3 & 3 \\
\hline$H_{18 e}$ & 18 & 6480 & $D_{8}$ & 4 & 6 \\
\hline$H_{18 f}$ & 18 & 6480 & $D_{8}$ & 5 & 6 \\
\hline
\end{tabular}

Table 3: The hyperovals of $H(3,4)$

We call two tuples $\bar{a}_{1}, \bar{a}_{2} \in \mathbb{F}_{2}^{6} \times \mathbb{F}_{4}^{9}$ equivalent if one of them is obtained from the other by successively applying the maps $\sigma_{1}, \sigma_{2}, \ldots, \sigma_{6}$ (described in Tables 1 and 2 ). GAP computations [11] show that there are 23 equivalence classes and that their sizes $N$ are as in column 3 of Table 3 . For each equivalence class, we have also determined a tuple of minimal possible weight $w$. Such a tuple (which needs not to be unique) can be found in the last column of Table 4 , while the value of $w$ itself can be found in the second last column of that table.

We have also determined all even sets and hyperovals of $H(3,4)$ in an alternative way. We have implemented in GAP a computer model of $H(3,4)$ along with a permutation representation of its automorphism group on the set of points. The even sets are precisely the sets of points whose characteristic vectors are orthogonal with the characteristic vectors of the lines. Using this fact, we were able to show that there are up to isomorphism 676 even sets and 23 hyperovals, see [11].

In Table 3, we also mentioned several properties of the hyperovals $H$ (obtained by 


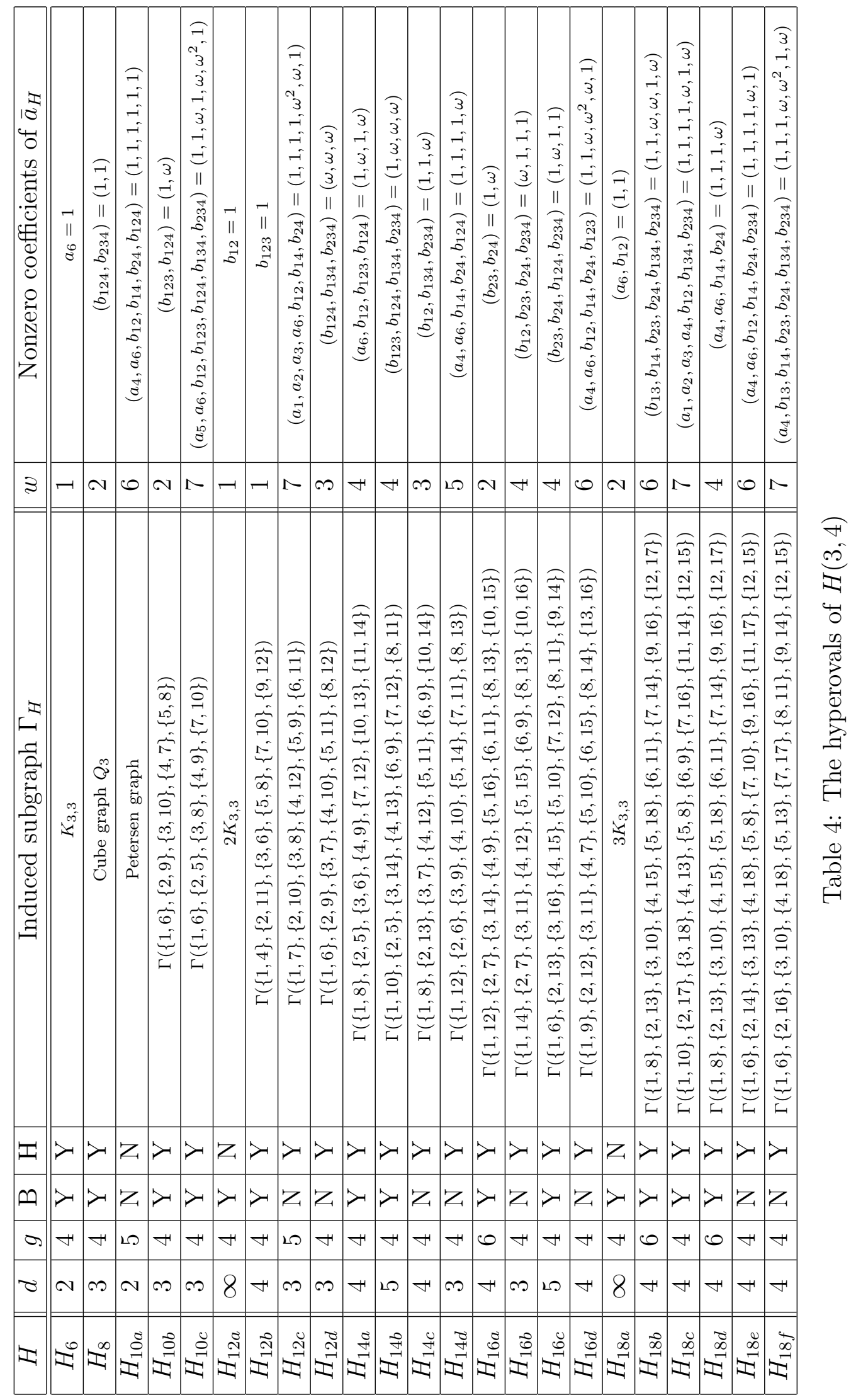


means of computations). This information includes the total number $v$ of points, the structure of the stabilizer $S$ of the hyperoval, the number $O_{1}$ of orbits of $S$ on the hyperoval and the number $\mathrm{O}_{2}$ of orbits of $S$ on its complement.

Suppose $P$ is a partition of some set $V=\{1,2, \ldots, 2 n\}$ with $n \in \mathbb{N} \backslash\{0,1\}$ in subsets of size 2 such that $|x-y| \notin\{1,2 n-1\}$ for every $\{x, y\}$ in $P$. Then the graph $\Gamma(P)$ with vertex set $V$ and edge set $P \cup\{\{1,2\},\{2,3\}, \ldots,\{2 n-1,2 n\},\{2 n, 1\}\}$ is a Hamiltonian regular graph of valency 3. Conversely, every 3-regular Hamiltonian graph on $2 n$ vertices is isomorphic to such a graph.

For each hyperoval $H$ in the classification, we have also implemented (with the aid of Sage [18]) the subgraph $\Gamma_{H}$ of the collinearity graph induced on $H$, see [11]. We verified that with exception of three cases, these graphs are all Hamiltonian (of degree 3) and so they are of the above-described form. A description of these graphs can be found in Table 4. The three non-Hamiltonian graphs are isomorphic to the Petersen graph and the unions $i K_{3,3}$ of $i \in\{2,3\}$ complete bipartite graphs of type $K_{3,3}$. Certain of the Hamiltonian graphs are known under a certain name: the graph $\Gamma_{H_{6}}$ is just $K_{3,3}$, the graph $\Gamma_{H_{8}}$ is the cube graph $Q_{3}$ of diameter 3 , the graph $\Gamma_{H_{10} c}$ is the Möbius ladder on 10 vertices and the graph $\Gamma_{H_{12 b}}$ is the prism graph $K_{2} \times C_{6}$ on 12 vertices. All the graphs mentioned in Table 4 are mutually nonisomorphic, with exception of $\Gamma_{H_{18 b}}$ and $\Gamma_{H_{18 d}}$. So, nonisomorphic hyperovals can have isomorphic induced subgraphs. Besides a construction, Table 4 list some other information about the graphs, like the diameter $d$, the girth $g$ and the answers B and $\mathrm{H}$ (being $\mathrm{Y}(\mathrm{es})$ or $\mathrm{N}(\mathrm{o})$ ) to the questions whether they are bipartite or Hamiltonian.

Our classification of the hyperovals shows that some 3-regular graphs on 12 and 14 vertices are missing in the discussion in [13, and others do not correspond to hyperovals. Our computations ([11]) show that the graphs depicted in Figures 11 and 14 of [13] do not correspond to hyperovals of $H(3,4)$. One of the two graphs depicted in Figure 10 of [13. does not correspond to a hyperoval of $H(3,4)$ while the other is isomorphic to $\Gamma_{H_{12 b}}$. The graphs depicted in Figures 7 and 12 of [13] are respectively isomorphic to $\Gamma_{H_{12 c}}$ and $\Gamma_{H_{14 a}}$. There seems to be no mentioning in [13] of the graphs $\Gamma_{H_{12 d}}, \Gamma_{H_{14 b}}, \Gamma_{H_{14 c}}$ and $\Gamma_{H_{14 d}}$.

Several hyperovals of $H(3,4)$ have already been described in the literature. We give an overview of these constructions and mention which hyperovals in the classification they correspond to. The various properties of the hyperovals mentioned in Tables 3 and 4 have helped in the identification process.

If $H$ is a hyperbolic line of $H(3,4)$, then $H \cup H^{\perp}$ is a hyperoval of $H(3,4)$ by [12, Theorem 2.1]. This hyperoval is isomorphic to $H_{6}$.

An ovoid of a generalized quadrangle is a set of points meeting each line in a singleton. If $O_{1}$ and $O_{2}$ are two distinct ovoids of a generalized quadrangle, then the symmetric difference $O_{1} \Delta O_{2}$ of $O_{1}$ and $O_{2}$ is a hyperoval by [12, Theorem 6.1]. By the main result of [1], we know that the generalized quadrangle $H(3,4)$ has up to isomorphism two ovoids: the classical ovoids arising by intersecting the underlying Hermitian variety with nontangent planes, and the nonclassical ovoids. Every nonclassical ovoid is of the form $(O \backslash H) \cup H^{\perp}$, where $O$ is a classical ovoid and $H$ is a hyperbolic line contained in $O$.

Computations show that the hyperovals that arise as symmetric differences of two 
classical ovoids are precisely the hyperovals isomorphic to $H_{12 a}$ and $H_{16 a}$. The hyperovals that arise as a symmetric difference of a classical and a nonclassical ovoid are precisely the hyperovals isomorphic to $H_{6}, H_{14 a}, H_{18 a}$ and $H_{18 d}$. The hyperovals that arise as symmetric differences of two nonclassical ovoids are precisely the hyperovals isomorphic to $H_{10 b}, H_{12 a}, H_{12 b}, H_{16 c}, H_{18 b}$ and $H_{18 c}$. By construction, the underlying graphs of these hyperovals are bipartite.

Let $\pi$ be a nontangent plane to the Hermitian surface $H\left(3, q^{2}\right)$ and let $h_{1}, h_{2}, \ldots, h_{n}$ be a nonempty collection of hyperbolic lines contained in $\pi$ such that every point of $\pi \cap H\left(3, q^{2}\right)$ is contained in at most two hyperbolic lines of the collection $h_{1}, h_{2}, \ldots, h_{n}$. Let $B$ denote the set of all points of $\pi \cap H\left(3, q^{2}\right)$ contained in precisely two hyperbolic lines of this collection. By [6, Theorem 2.2], we then know that the set $H:=\left(h_{1} \cup h_{2} \cup\right.$ $\left.\cdots \cup h_{n} \cup h_{1}^{\perp} \cup h_{2}^{\perp} \cup \cdots \cup h_{n}^{\perp}\right) \backslash B$ is a hyperoval of size $2(n(q+1)-|B|)$ of $H\left(3, q^{2}\right)$. The special cases of this construction where either $B=\emptyset$ or $B=h_{1} \cup h_{2} \cup \cdots \cup h_{n}$ were also discussed in Pavese [16] (Examples 1 and 2). Our computations show that the hyperovals of $H(3,4)$ that can be obtained in this way are the hyperovals isomorphic to $H_{6}, H_{10 b}$, $H_{12 a}, H_{12 c}, H_{14 a}, H_{14 c}, H_{16 a}, H_{18 a}, H_{18 b}, H_{18 c}$ or $H_{18 d}$.

Let us now mention the remaining constructions from the literature for hyperovals of $H\left(3, q^{2}\right)$. In [4], a hyperoval of size $2\left(q^{3}-q\right)$ of $H\left(3, q^{2}\right), q$ even, was constructed which has its full automorphism group isomorphic to $P S L(2, q) \times C_{2} \times C_{2}$. For $q=2$, this hyperoval is isomorphic to $H_{12 b}$. In [5], a hyperoval of size $2\left(q^{2}+1\right)$ of $H\left(3, q^{2}\right), q$ even, was constructed whose full automorphism group is a group of type $D_{4\left(q^{2}+1\right)}, D_{2\left(q^{2}+1\right)}$ or $C_{q^{2}+1} \rtimes C_{4}$. For $q=2$, this hyperoval is isomorphic to $H_{10 c}$. In [6, Proposition 5.1], a hyperoval of size $q^{2}+2 q$ of $H\left(3, q^{2}\right), q$ even, was constructed. For $q=2$, this hyperoval is isomorphic to $H_{8}$.

\section{Acknowledgement}

The second author, Mou Gao, is supported by the State Scholarship Fund (File No. 201806065052) and the National Natural Science Foundation of China (grant number $71771035)$.

\section{References}

[1] A. E. Brouwer and H. A. Wilbrink. Ovoids and fans in the generalized quadrangle Q(4,2). Geom. Dedicata 36 (1990), 121-124.

[2] F. Buekenhout and X. Hubaut. Locally polar spaces and related rank 3 groups. J. Algebra 45 (1977), 391-434.

[3] P. J. Cameron, D. R. Hughes and A. Pasini. Extended generalized quadrangles. Geom. Dedicata 35 (1990), 193-228.

[4] A. Cossidente, O. H. King and G. Marino. Hyperovals of $H\left(3, q^{2}\right)$ when $q$ is even. $J$. Combin. Theory Ser. A 120 (2013), 1131-1140. 
[5] A. Cossidente, O. H. King and G. Marino. Hyperovals arising from a Singer group action on $H\left(3, q^{2}\right), q$ even. Adv. Geom. 16 (2016), 481-486.

[6] A. Cossidente and F. Pavese. Hyperoval constructions on the Hermitian surface. Finite Fields Appl. 25 (2014), 19-25.

[7] B. De Bruyn. On the Grassmann modules for the unitary groups. Linear Multilinear Algebra 58 (2010), 887-902.

[8] B. De Bruyn. The pseudo-hyperplanes and homogeneous pseudo-embeddings of AG $(n, 4)$ and PG(n,4). Des. Codes Cryptogr. 65 (2012), 127-156.

[9] B. De Bruyn. Pseudo-embeddings and pseudo-hyperplanes. Adv. Geom. 13 (2013), $71-95$.

[10] B. De Bruyn. The pseudo-hyperplanes and homogeneous pseudo-embeddings of the generalized quadrangles of order $(3, t)$. Des. Codes Cryptogr. 68 (2013), 259-284.

[11] B. De Bruyn and M. Gao. Computer computations for "The homogeneous pseudoembeddings and hyperovals of the generalized quadrangle $H(3,4)$ ". Available online from http://cage. ugent. be/geometry/preprints.php.

[12] A. Del Fra, D. Ghinelli and S. E. Payne. $(0, n)$-sets in a generalized quadrangle. pp. 139-157 in "Combinatorics '90 (Gaeta, 1990)", Ann. Discrete Math. 52, NorthHolland, 1992.

[13] A. A. Makhnev. Extensions of $G Q(4,2)$, the description of hyperovals. Discrete Math. Appl. 7 (1997), 419-435.

[14] D. V. Pasechnik. The triangular extensions of a generalized quadrangle of order $(3,3)$. Bull. Belg. Math. Soc. Simon Stevin 2 (1995), 509-518.

[15] D. V. Pasechnik. The extensions of the generalized quadrangle of order $(3,9)$. European J. Combin. 17 (1996), 751-755.

[16] F. Pavese. Hyperovals on $H\left(3, q^{2}\right)$ left invariant by a group of order $6(q+1)^{3}$. Discrete Math. 313 (2013), 1543-1546.

[17] S. E. Payne and J. A. Thas. Finite generalized quadrangles. Second edition. EMS Series of Lectures in Mathematics. European Mathematical Society (EMS), 2009.

[18] Sage Mathematics Software (Version 6.3), The Sage Developers, 2014, http://www. sagemath.org.

[19] The GAP Group, GAP - Groups, Algorithms, and Programming, Version 4.7.5, 2014. (http://www.gap-system.org) 


\section{Addresses of the authors:}

Ghent University

Department of Mathematics: Algebra and Geometry

Krijgslaan 281 (S25), B-9000 Gent, Belgium

Email: Bart.DeBruyn@Ugent.be, Gao.Mou@UGent.be 